\title{
Long-time experimental investigation of turbulent superstructures in Rayleigh-Bénard convection by noninvasive simultaneous measurements of temperature and velocity fields
}

\author{
Sebastian Moller ${ }^{1}$ (D) $\cdot$ Christian Resagk $^{1} \cdot$ Christian Cierpka $^{1}$
}

Received: 10 July 2020 / Revised: 21 November 2020 / Accepted: 30 November 2020

(C) The Author(s) 2021

\begin{abstract}
Large-scale mean patterns in Rayleigh-Bénard convection, also referred to as turbulent superstructures, have mainly been studied by means of numerical simulations so far, but experimental investigations are still rare. However, the analysis of turbulent superstructures, which are of great importance due to their effect on the local transport of heat and momentum, require both numerical and experimental data. Therefore, within the scope of this study measurements were performed in the horizontal mid plane and in a horizontal plane closer to the top of a Rayleigh-Bénard cell with an aspect ratio of $\Gamma=l / h=25$, thereby showing the initial formation of turbulent superstructures and their long-time rearrangement. The turbulent superstructures are investigated experimentally by noninvasive simultaneous measurements of temperature and velocity fields, using the color signal of thermochromic liquid crystals (TLCs) for the evaluation of the temperature and their temporal displacement for the determination of all three velocity components in the measurement planes via stereoscopic particle image velocimetry (stereo-PIV). Applying this measuring technique it is demonstrated that the time-averaging of instantaneous temperature and velocity fields uncovers the turbulent superstructures in both fields. Furthermore, the combination of the temperature and velocity data is used to characterize the local heat flux quantified by the local Nusselt number, which confirms that the turbulent superstructures strongly enhance the heat transfer in Rayleigh-Bénard convection.
\end{abstract}

\section{Graphic abstract}
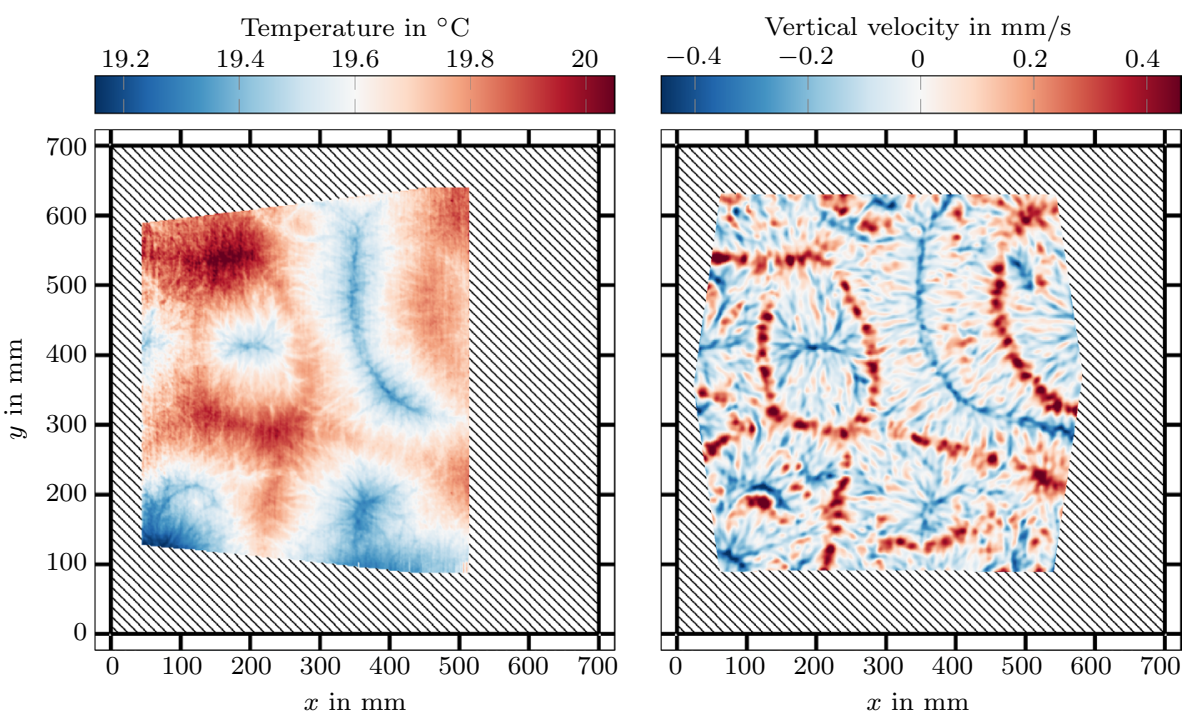

Sebastian Moller

sebastian.moller@tu-ilmenau.de

Extended author information available on the last page of the article 


\section{Introduction}

Since the flow mechanisms in thermally driven convection are of importance for many technical applications, such as the steel casting (Burr and Müller 2001), crystal growth (Cramer et al. 2013) and the ventilation of passenger compartments in trains or airplanes (Zhang et al. 2020), but also for a better understanding of geo- and astrophysical settings (Schumacher and Sreenivasan 2020), the detailed analysis of this type of flow has become increasingly important in the last decades. In order to study the flow mechanisms by means of laboratory experiments or numerical simulations, the so-called Rayleigh-Bénard setup has established as an appropriate model. This model consists of a fluid volume enclosed by adiabatic side walls, which is uniformly heated from below and cooled from above. Applying Rayleigh-Bénard cells with flat surfaces, the boundary conditions of complex technical and natural systems with thermal buoyancy as the main flow driving force are simplified. However, this model allows to investigate typical characteristics of thermally driven flows and is therefore suited to gain deeper insights into these systems. For example, the flow mechanisms in the earth's atmosphere and in the oceans can be better understood based on investigations of the flow in a Rayleigh-Bénard setup, thereby helping to improve the weather forecast. Therefore, the flow inside this setup, i.e. Rayleigh-Bénard convection, has already been analyzed comprehensively (Bodenschatz et al. 2000).

Flow structures in Rayleigh-Bénard convection cover a large range of different length and time scales. On the one hand, flow structures on very small scales may appear, especially when the flow is highly turbulent (Emran and Schumacher 2008; Lohse and Xia 2010; Scheel et al. 2013). On the other hand, for example in Rayleigh-Bénard cells with a similar characteristic length $l$ and height $h$, i.e. with aspect ratio $\Gamma=l / h \approx 1$, the well-known large scale circulation (LSC) roll spanning nearly the whole vertical cross-section of the Rayleigh-Bénard cell can be observed (Zhou et al. 2009; Mishra et al. 2010). Depending on the aspect ratio, this roll might also be divided and other states beside the one-roll state such as horizontally or vertically stacked rolls are possible (du Puits et al. 2007; van der Poel et al. 2012; Emran and Schumacher 2015). However, when the horizontal dimension of the Rayleigh-Bénard cell is considerably larger than its height, those clearly separated rolls cannot be found anymore. Thus, the flow inside cells with very large aspect ratios, which are relevant to study effects in the atmosphere, seems to be less organized at the first glance. Averaging the flow field over time shows, however, that there is also a clear organization in this case, as some larger structures dominating the flow inside the cell are revealed, for example clearly illustrated by Bailon-Cuba et al. (2010) and Pandey et al. (2018). The flow structures in Rayleigh-Bénard convection, which become observable after time-averaging and are also referred to as turbulent superstructures, are currently widely addressed, since those represent the global flow dynamics and are of great relevance for the transfer of heat and momentum.

With respect to Rayleigh-Bénard convection, turbulent superstructures have mainly been investigated by means of numerical simulations so far. Even though those flow structures have not been termed like this in earlier studies, they have already been reported and discussed for different input parameters of the simulations, which determine the Rayleigh-Bénard flow. In this context, the geometry of the simulation domain, the properties of the fluid and the thermal driving force of the flow have been varied by applying different aspect ratios, Prandtl numbers and Rayleigh numbers, respectively. For example, Hartlep et al. (2003) investigated the typical size of large-scale structures in Rayleigh-Bénard convection for Rayleigh numbers ranging from the onset of convection up to about $\mathrm{Ra} \approx 10^{7}$ as well as for Prandtl numbers in the range approximately limited by $\operatorname{Pr} \in[0.3,100]$, using a simulation domain with an aspect ratio of $\Gamma=10$ in most cases. Hartlep et al. (2005) also presented an overview of the corresponding large-scale convection patterns and further results with regard to different types of mean flow components and the convective heat transport. In order to clarify also the effect of the aspect ratio of the simulation domain on the large-scale patterns, this parameter was varied in later studies by von Hardenberg et al. (2008), Bailon-Cuba et al. (2010) and Stevens et al. (2018). Furthermore, Emran and Schumacher (2015) investigated the drift of large-scale patterns in Rayleigh-Bénard convection and additionally checked their robustness to side wall effects. By analyzing the time-averaged temperature fields of successive time intervals, the drift of large-scale patterns was also shown by Sakievich et al. (2016).

Later, in the study of Pandey et al. (2018), in which the large scale-scale patterns have now been termed turbulent superstructures, those have been presented in dependency of the Rayleigh number and Prandtl number for aspect ratio $\Gamma=25$. In comparison with Hartlep et al. (2005), the Rayleigh number was also varied up to $\mathrm{Ra}=10^{7}$, but the range of the Prandtl number was considerably extended towards lower values of $\operatorname{Pr} \ll 0.1$, which requires massively parallel supercomputing. Furthermore, Pandey et al. (2018) identified characteristic time and length scales that separate fast turbulent fluctuations on small scales from the slowly evolving turbulent superstructures on larger scales. Moreover, alternative novel approaches for the investigation of turbulent superstructures are discussed in further studies, such as using Lagrangian trajectory clusters (Schneide et al. 2018) 
and deep-learning algorithms (Fonda et al. 2019). Most recently, Krug et al. (2020) analyzed turbulent superstructures based on the fluctuation fields of the temperature and the vertical velocity for $10^{5} \leq \mathrm{Ra} \leq 10^{9}$ at fixed $\operatorname{Pr}=1$ and $\Gamma=32$, thereby explicitly pointing out that superstructures of the same size exist in both fields, contrary to the findings of prior studies. Finally, Green et al. (2020) studied the interplay between small-scale fluctuations and turbulent superstructures by means of resolved energy budgets for $10^{4} \leq \mathrm{Ra} \leq 10^{8}$, also applying a fixed $\operatorname{Pr}=1$ and $\Gamma=24$.

All the studies regarding turbulent superstructures outlined above are based on numerical simulations. In contrast, results obtained from experimental investigations have, as far as the authors know, rarely been published so far. However, especially experiments offer the possibility to capture the slowly evolving reorganization of turbulent superstructures over several hours and are therefore complementary to spatially highly resolved numerical data, which are not available for such extended time periods. Some exemplary velocity measurements via particle image velocimetry (Raffel et al. 2018), which show the existence of turbulent superstructures in Rayleigh-Bénard convection, were performed by Cierpka et al. (2019) in the so-called SCALEX facility (Kästner et al. 2018). For these measurements pressurized air as well as sulfur-hexafluoride $\left(\mathrm{SF}_{6}\right)$ were used as the working fluid in a Rayleigh-Bénard cell with $\Gamma=10$, thereby achieving a Rayleigh number of $\mathrm{Ra}=5 \times 10^{5}$. However, since those investigations were not primarily focused on turbulent superstructures and to the best of the authors' knowledge other experimental analysis are not available yet, the current study is intended to investigate turbulent superstructures from the experimental point of view more in detail. For this, simultaneous measurements of the temperature and velocity field in large aspect ratio Rayleigh-Bénard convection using so-called thermochromic liquid crystals (TLCs) are performed. After the following explanation of the experimental setup and the measurement technique in Sect. 2, the results of the measurements are presented in Sect. 3. On the basis of the results, a conclusion follows in Sect. 4 and finally an outlook is given in Sect. 5 .

\section{Experimental setup and methods}

In order to study turbulent superstructures in Rayleigh-Bénard convection, a Rayleigh-Bénard cell with dimensions of $l \times w \times h=700 \mathrm{~mm} \times 700 \mathrm{~mm} \times 28 \mathrm{~mm}$ and thus an aspect ratio of $\Gamma=l / h=25$ has been set up. Using this cell, turbulent superstructures can be observed in a large field of view, while it can still be handled under common laboratory conditions. Furthermore, the range of the Rayleigh numbers, which can be achieved with this cell, was a very important aspect during its design, since this dimensionless number determines the thermal driving force of the flow in the cell. The Rayleigh number is defined as $\mathrm{Ra}=\alpha g \Delta T h^{3} /(v \kappa)$ with the thermal expansion coefficient $\alpha$, acceleration due to gravity $g$, kinematic viscosity $v$, thermal diffusivity $\kappa$ and the temperature difference $\Delta T$ between the heating plate at the bottom and the cooling plate at the top. For the present study, water is used as the working fluid in the cell with $\Gamma=25$, providing the possibility to achieve Rayleigh numbers in the range $10^{5} \leq \mathrm{Ra} \leq 5 \times 10^{6}$ by applying moderate temperature differences up to $\Delta T=10 \mathrm{~K}$. As it can be seen in the following, the mean temperature of the water in the cell is about $T=20^{\circ} \mathrm{C}$ for the presented measurements, such that a Prandtl number of $\operatorname{Pr}=v / \kappa \approx 7$ can be assumed. This mean temperature was also adjusted in the lab environment, thereby minimizing the heat flux across the side walls of the cell, which may affect the flow in the Rayleigh-Bénard cell. However, for a cell with a large aspect ratio as in this case, the influence of the side walls is usually less crucial a priori, as the main part of the fluid is far away from the lateral boundaries.

Here, long-time measurements are performed in horizontal planes of the cell, since those allow to observe the footprint of turbulent superstructures in Rayleigh-Bénard convection most clearly. However, when analyzing turbulent superstructures, the measurements should cover a large fraction of the horizontal planes, which is very challenging from the experimental point of view. If optical measurement techniques are applied, this must be realized by using either a transparent heating or cooling plate (Kästner et al. 2018). In the present case, the cooling plate is made of glass and cooled by water flowing above it in an external circuit, indicated in Fig. 1, which shows a schematic sketch of the experiment and a photograph of the real experimental setup. In order to ensure a safe operating mode of the cooling plate, i.e. to prevent the breakage of the glass, particular emphasis was given to a low pressure drop in the design of the cooling water circuit. The heating plate is made of aluminum and also flown through by water within a second external circuit for controlling its temperature. Furthermore, the side wall is made of glass, enabling full optical access to the whole Rayleigh-Bénard cell. As can be seen on the right side of Fig. 1, the experimental setup is covered by insulating material. On the one hand, this is advantageous for a homogeneous temperature distribution on the isothermal plates. On the other hand, the insulation is also of advantage for the boundary conditions at the side walls of the cell. Even though the surrounding temperature is adapted to the mean temperature of the water in the cell and thus only a small heat flux across the side walls may occur, this heat flux is further lowered by an insulation, which has been removed from the wall for a better illustration in Fig. 1.

During the measurements, a light sheet is used to illuminate small particles dispersed in the cell through one of 

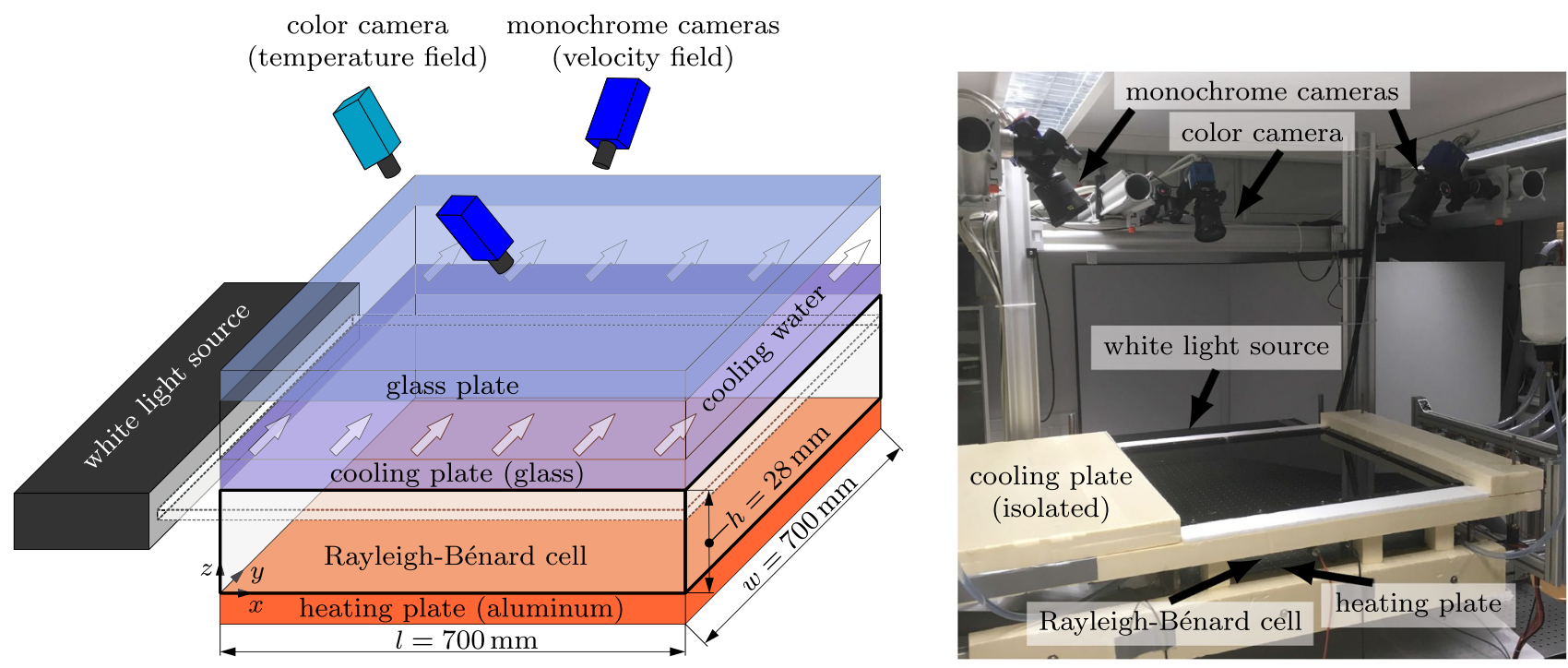

Fig. 1 Sketch of the experiment (left) and a photograph of the real experimental setup (right) for simultaneous velocity and temperature field measurements in horizontal planes of the Rayleigh-Bénard (RB) cell. The photograph shows a Rayleigh-Bénard cell with aspect ratio $\Gamma=10$ for better visibility, but for the current study a cell with $\Gamma=25$ was used. It should be noted that the coordinate system in the left corner of the sketch is valid for all of the following investigations

a Gaussian distribution are applied. The TLCs of this type nominally start to get red at a temperature of $T=20^{\circ} \mathrm{C}$ and change their color across the visible wavelength spectrum until they appear blue at $T=40^{\circ} \mathrm{C}$. However, these specifications given by the manufacturer only apply to the case that the TLCs are illuminated and observed from the same direction. Here, the color signal reflected by the TLCs within the light sheet is recorded with a color camera (sCMOS pco edge 5.5 color, PCO AG), which can be seen in Fig. 1, using an observation angle of about $\varphi_{\mathrm{cc}}=65^{\circ}$ between the axis of the color camera and the light sheet. Compared to the nominal specifications of the TLCs valid for $\varphi_{\mathrm{cc}}=0^{\circ}$, the effective temperature measuring range drastically decreases when increasing the observation angle of the TLCs up to $\varphi_{\mathrm{cc}}=90^{\circ}$, since the main color change from red to blue passes within a considerably smaller temperature range. Seeming to be disadvantageous, this feature turns out to be very useful in some cases, when the observation angle can be adapted such that the temperatures in the range of interest can be measured with a lower uncertainty (Moller et al. 2019). However, in the present study a fixed temperature range of interest for the long-time convection measurements presented in Sect. 3.3 was not given a priori. Therefore, the observation angle and the temperature difference between the heating and cooling plate could be adapted in a way that allows to precisely measure the occurring temperatures on the one hand and to clearly observe turbulent superstructures on the other hand.

In order to demonstrate that the turbulent superstructures span the whole height of the Rayleigh-Bénard cell, 
the measurements were performed in two different horizontal planes, with one being at mid-height and another in closer vicinity to the cooling plate. The center of the light sheet was located at $z=14 \mathrm{~mm}(z / h=0.5)$ and $z=24 \mathrm{~mm}$ $(z / h \approx 0.86)$, respectively, when measured from the surface of the heating plate. Even though the top measurement plane is close to the cooling plate, the distance has been chosen large enough, such that the plane is outside the thermal boundary layer and accordingly the occurring temperatures fluctuate around the average temperature of the heating and cooling plate. Therefore, the temperature measuring range can be better estimated and adapted to the range of the color play of the TLCs. In both planes the measurements have been performed with the same temperature settings at the thermostatic baths, which are used to drive the flow through the heating and cooling plate and to control their temperature. The temperatures of the plates are listed in Table 1 and have been determined based on measurements with several small PT100 sensors, considering the whole measurement period in each plane, respectively. For both the heating and cooling plate the differences of the local temperatures on their surface measured with the sensors did not exceed $0.1 \mathrm{~K}$ in this case, thereby ensuring that the isothermal boundary condition is fulfilled in a good approximation and the flow in the cell is not determined by temperature inhomogeneities on the plates. Furthermore, the resulting Rayleigh number and the free-fall time $t_{\mathrm{f}}=\sqrt{h /(\alpha g \Delta T)}$, which represents a typical convective time-scale, can also be seen in Table 1 . The free-fall times are considerably larger than the thermal response time of the TLCs, which is in the order of milliseconds (Dabiri 2008). Therefore, the temporal delay of the temperature measurements can be neglected, confirming that the TLCs are well suited to represent the local temperatures in this case. Moreover, it should be noted that the density of the TLCs nearly matches that of water in the given temperature range, such that the velocity of sedimentation $u_{\mathrm{s}}$ is very small. The comparison with the free-fall velocity $u_{\mathrm{f}}=h / t_{\mathrm{f}}$ yields $u_{\mathrm{s}} \approx 10^{-4} u_{\mathrm{f}}$, ensuring that sedimentation is negligible, since the typical velocities occurring in the Rayleigh-Bénard flow are considerably larger as it will be shown in Sect. 3.3.

For the temperature measurements via the color signal of TLCs, calibration measurements must be performed, yielding the correlation between the color and temperature. The details of the calibration procedure can be found in Moller et al. (2020). However, the main principle of the calibration is also obvious from Figs. 2 and 3. During the calibration, 100 images of the TLCs are taken for different isothermal states in the Rayleigh-Bénard cell, exemplarily depicted for $T=19.7^{\circ} \mathrm{C}$ of the top plane measurement in Fig. 2a. This image clearly shows that the color shade of TLCs does not only depend on the temperature, but also on the observation angle, which may considerably vary within the camera's field of view (Moller et al. 2019, 2020). Hence, the calibration is performed locally, meaning the whole field of view is split into many interrogation windows and the color shade of the TLCs is extracted locally in dependency of the temperature. The single interrogation windows consist of $16 \times 16$ pixels in this study, corresponding to the physical dimensions given in Table 1, and are overlapped by $50 \%$ in each case. This size of the interrogation windows is used for both the calibration measurements and the temperature measurements in Rayleigh-Bénard convection.

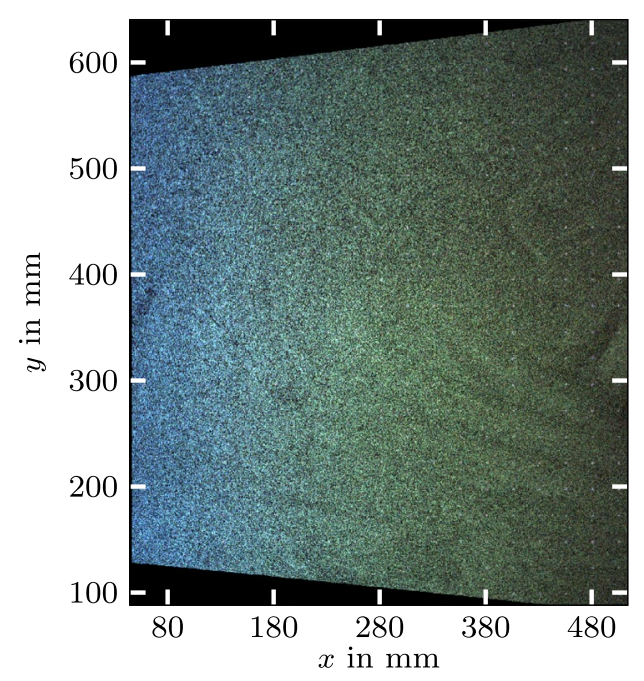

(a) TLCs at $T=19.7^{\circ} \mathrm{C}$

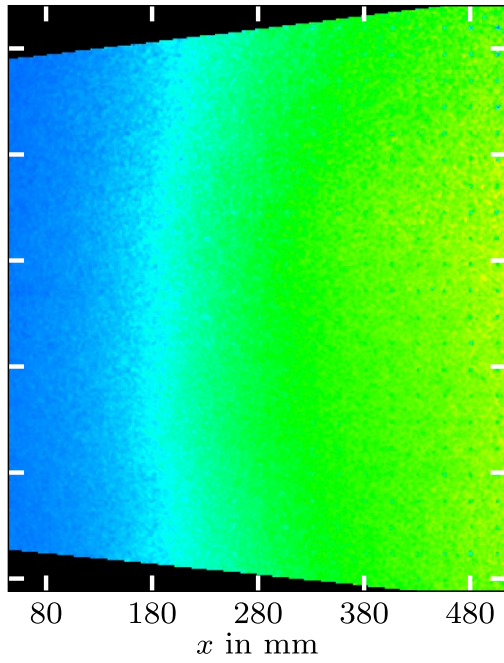

(b) Extracting the color shade

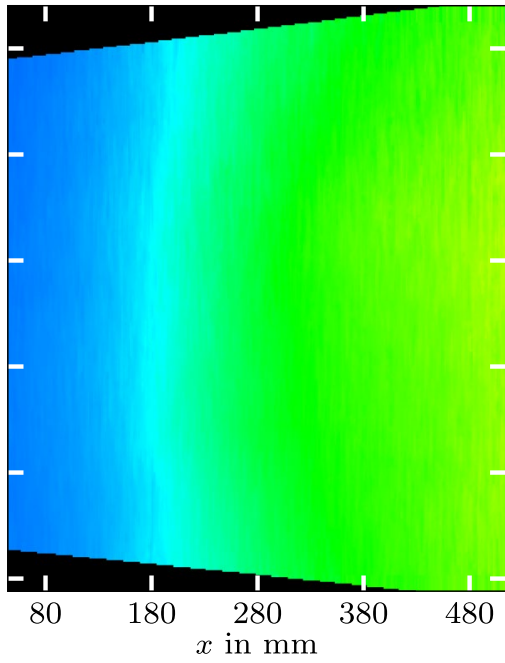

(c) Median filtering in $y$-direction

Fig. 2 Processing of the images of thermochromic liquid crystals for the calibration of the temperature measurements at the example of the temperature level $T=19.7^{\circ} \mathrm{C}$ of the top plane measurement 


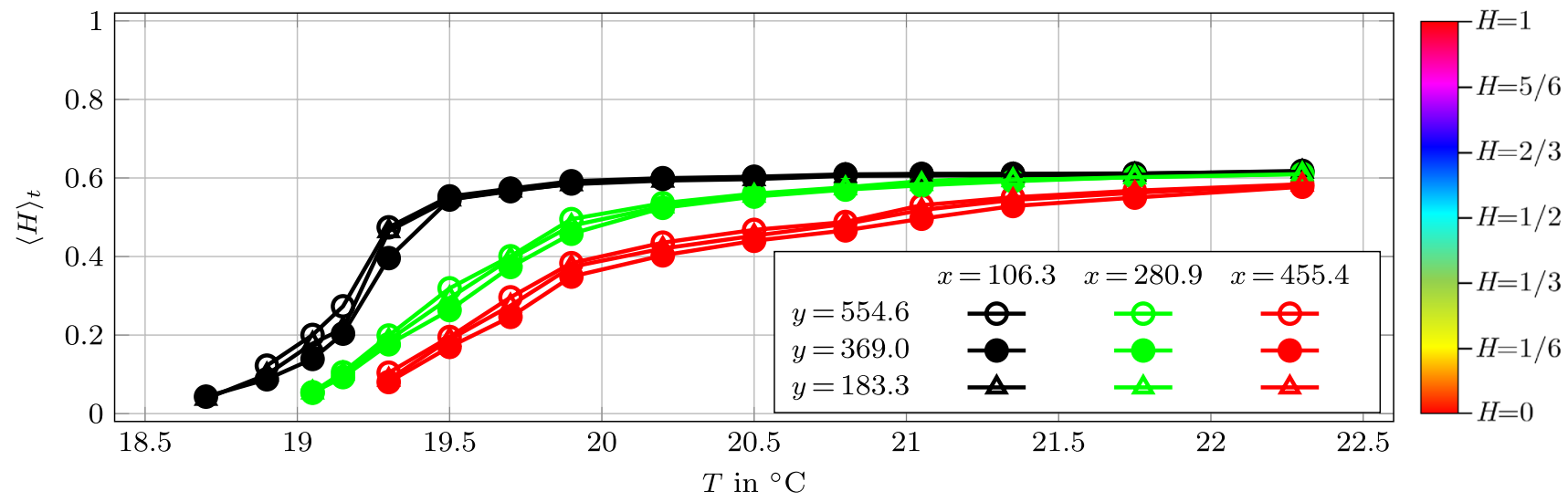

(a) Calibration curves for different positions within the field of view given in millimeters

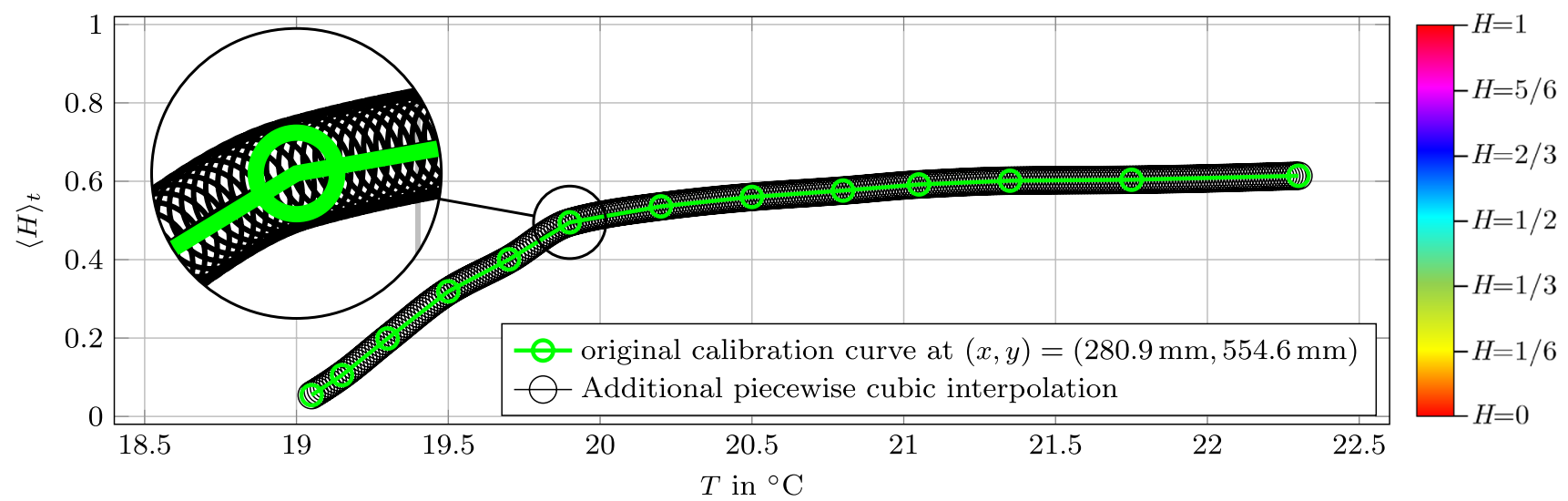

(b) Effect of piecewise cubic interpolation applied to an exemplary original calibration curve

Fig. 3 Exemplary calibration curves showing the correlation between the color of the TLCs R20C20W in terms of the hue value and the temperature. The color bars on the right side of the figures illustrate the color corresponding to the hue values, respectively

Table 1 Settings for the measurements presented in Sect. 3.3

\begin{tabular}{|c|c|c|c|c|c|c|c|c|}
\hline Plane & $z(z / h)$ & $T_{\mathrm{h}}$ & $T_{\mathrm{c}}$ & $\mathrm{Ra}$ & $t_{\mathrm{f}}$ & FOV & WIW-T (OL) & WIW-U (OL) \\
\hline Top & 24 mm (0.86) & $20.89^{\circ} \mathrm{C}$ & $18.38^{\circ} \mathrm{C}$ & $7.54 \times 10^{5}$ & $2.37 \mathrm{~s}$ & $459 \mathrm{~mm} \times 466 \mathrm{~mm}$ & $3.2 \mathrm{~mm}(50 \%)$ & $4.8 \mathrm{~mm}(50 \%)$ \\
\hline Mid & $14 \mathrm{~mm}(0.5)$ & $20.90^{\circ} \mathrm{C}$ & $18.34^{\circ} \mathrm{C}$ & $7.65 \times 10^{5}$ & $2.35 \mathrm{~s}$ & $460 \mathrm{~mm} \times 470 \mathrm{~mm}$ & $3.2 \mathrm{~mm}(50 \%)$ & $4.8 \mathrm{~mm}(50 \%)$ \\
\hline
\end{tabular}

Starting from the second column, the Table lists the position of the measurement plane $z$, the temperature of the heating plate $T_{\mathrm{h}}$ and cooling plate $T_{\mathrm{c}}$, the Rayleigh number Ra, the free-fall time $t_{\mathrm{f}}$, the size of the field of view FOV as well as the width of the interrogation windows for the evaluation of the temperature field WIW-T and of the velocity field WIW-U. The overlap OL of the interrogation windows is given in the brackets of the last two columns, respectively

On the basis of the TLC images the so-called hue value, which represents their color shade, is determined for each interrogation window and temperature step of the calibration (Moller et al. 2020). For illustration the color shade corresponding to the local hue values of the TLCs in Fig. 2a is depicted in Fig. 2b. In this figure some local outliers become apparent, for example caused by the pattern of white dots printed on the heating plate, which is part of the calibration regarding the dimensions of the field of view and can especially be seen on the right side of Fig. 2a. In order to overcome this issue, a sliding median filter is applied to the local hue values, taking into account the values of the neighboring 10 interrogation windows in positive and negative $y$-direction, thereby not smoothing out the distinctive physically relevant trend of the hue value in the $x$-direction. The result in Fig. 2c clearly shows the effect of the sliding median filter, since the local outliers are removed. This confirms the usefulness of the filter to compensate for 
local defects in the calibration measurements and to make the measuring technique more robust. However, this sliding median filter is not used for the convection measurements, which feature temperature gradients in all directions. Therefore, the main source of local outliers, which is the pattern of dots in the background, is then taken into account by masking the points and using the temperatures of the adjacent interrogation windows for interpolation.

Even though the focus in this study is not on the calibration measurements and the uncertainty of the temperature measurements using the color signal of TLCs, the most important aspects are shortly discussed by means of the Fig. 3a. This figure shows the color of the TLCs in terms of the hue value depending on the temperature for nine different interrogation windows distributed nearly over the whole field of view, resulting in nine different local calibration curves. For each temperature step the local hue values were averaged temporally over the 100 image recordings, indicated by the notation $\langle H\rangle_{t}$. As it could already be expected from Fig. 2, the calibration curves in Fig. 3a demonstrate that there is a clear color trend when varying the position of the interrogation windows along the $x$-axis and, therefore, along the direction of the incidence of light and the inclination of the color camera. The calibration curves also confirm that the color trend along the $y$-axis can be neglected. Furthermore, the hue value mostly varies in between $0 \leq\langle H\rangle_{t} \leq 0.6$, corresponding to a color change from red to blue with increasing temperature. Since the transition from red to blue as well as the local red start temperature of the TLCs strongly varies along the $x$-axis, the necessity of the local calibration approach is confirmed.

In order to determine the temperature fields of the following convection measurements, linear interpolation of the hue values in the local calibration curves is used within the scope of this study. However, as the slope of the calibration curves does not change continuously due to the discreteness of the calibration points, a piecewise cubic interpolation is applied to each local calibration curve, yielding additional calibration points in steps of $0.01 \mathrm{~K}$ along the entire curve. Therefore, the determination of the temperature is even more reliable, as the change of the slope between neighboring points within the local calibration curves is smoothed, which can exemplarily be seen in Fig. 3b. Using this method, a mean measurement uncertainty of about $0.1 \mathrm{~K}$ can be achieved, considering the whole temperature range of the calibration measurement and the whole field of view of the color camera. For detailed information about the uncertainty of the temperature measurement and alternative calibration approaches, e.g. based on neural networks, the interested reader is referred to Moller et al. (2020).

As already mentioned above, the two monochrome cameras are applied to simultaneously measure the velocity field based on the temporal displacement of the TLCs within the white light sheet. Here, both monochrome cameras are arranged in a plane parallel to the $y$-z-plane with an angle of about $\varphi_{\mathrm{mc}}=35^{\circ}$ between the optical axis of the camera and the vertical axis, respectively. The spatial resolution of those measurements was chosen such that the smallest convective flow structures can be resolved while complying with the general recommendations for particle image velocimetry measurements (Raffel et al. 2018). Hence, interrogation windows with $24 \times 24$ pixels and an overlap of $50 \%$ were used for the velocity measurements. The resulting physical dimensions of the interrogation windows are given in Table 1. Using these settings, the velocity components can be determined with a low uncertainty of measurement that amounts to a mean relative error of about $2 \%$, considering both the horizontal and vertical velocity components. For the temperature and velocity measurements all of the three cameras are equipped with wide angle objective lenses (Zeiss Otus 1.4/28) and tilt adapters for Scheimpflug correction, so that a large field of view can be imaged sharply. The area of the largest possible rectangle extracted from the intersection of the three cameras, which can be used for the evaluation of both the temperature and velocity field in Sect. 3.3, approximately covers $l_{x} \times l_{y}=16.4 \mathrm{~h} \times 16.7 \mathrm{~h}$.

In order to transfer the camera images into physical dimensions, a pattern of equidistant dots within the Rayleigh-Bénard cell was recorded with all the three cameras in two different horizontal planes. One of the planes is the surface of the heating plate with the pattern of dots directly printed on it. The images in the second horizontal plane were obtained from an additional flat calibration target that has the same pattern of the equidistant dots printed on its surface and was temporarily placed on the heating plate within the cell. However, as the temperature and velocity field measurements presented in this work were performed in the mid plane and in a plane closer to the top of the cell, the calibration had to be adapted. For this, the established stereoscopic self-calibration (Wieneke 2005), which is a common tool used to correct the displacement between the measurement and calibration plane, was applied in each case.

\section{Experimental results}

\subsection{Initial formation of turbulent superstructures}

In many experiments seeding particles for the investigation of the flow are inserted when the boundary conditions are already stationary, especially when the process of reaching the steady-state conditions takes a long time. Thus, the disturbance induced by the inserting of the particles temporarily affects the flow. Also in the presented experiment, reaching constant temperatures at the isothermal plates is a longlasting process, which is why the TLCs are inserted when the thermal boundary conditions are stationary. Therefore, 
prior to the long-time measurements of Rayleigh-Bénard convection for the investigation of turbulent superstructures, a preliminary experiment was performed, in order to study the initial development of the flow structures subsequent to an external disturbance. The disturbance after reaching the steady-state boundary conditions was induced via stirring of the fluid with slight pressure impulses of an external pump connected to a small hole near an edge of the side wall, which is also used for inserting the TLCs into the cell.

The initial flow state caused by the stirring is quickly extenuated and many flow structures due to thermal convection start to evolve, thereby also enabling to observe their initial development over time. It is emphasized that the specific arrangement of the flow structures immediately after the stirring is not replicable, as it depends on the initial conditions, i.e. on the arrangement of the flow structures before the stirring and also on the stirring process itself. However, the initial formation of the flow structures was analyzed in several independent runs of the experiment, where a gradual growth of the flow structures over time could be observed, respectively. As the progress of the growing was very similar in each case, it deserves closer attention and is exemplarily demonstrated in the following.

In order to show this development of the flow structures, which are directly correlated to the temperature field, only the color camera was applied to determine the temperature field for different points in time. The measurement was performed in the top plane with nearly the same temperatures at the heating and cooling plate as in the long-time measurements according to Table 1. The time instants of the successive temperature fields shown in Fig. 4 are given in seconds and in units of free-fall times $\tilde{t}=t / t_{\mathrm{f}}$, starting from the end of the stirring process at $\tilde{t}=0$, respectively.

Due to the stirring process, the temperature field at $t=0 \mathrm{~s}$ in Fig. 4a does not show any regular patterns. However, already in the temperature field at $t=100 \mathrm{~s}$ depicted in Fig. $4 \mathrm{~b}$ small regular patterns become apparent. Those patterns further assemble over time, which is especially obvious from $t=100 \mathrm{~s}$ to $t=500 \mathrm{~s}$ in the Fig. $4 \mathrm{~b}-\mathrm{d}$ and is also consistent with the findings based on numerical simulations by von Hardenberg et al. (2008). In the course of time, however, the typical pattern size from the global view does not considerably change anymore, shown by the temperature fields in the bottom row of Fig. 4. In those instantaneous temperature fields in the Fig. $4 \mathrm{e}-\mathrm{h}$ some outstanding larger patterns of warm and cold fluid can already be identified, indicating the footprint of the turbulent superstructures.

In order to further confirm the initial growth of the flow structures, the instantaneous temperature fields were

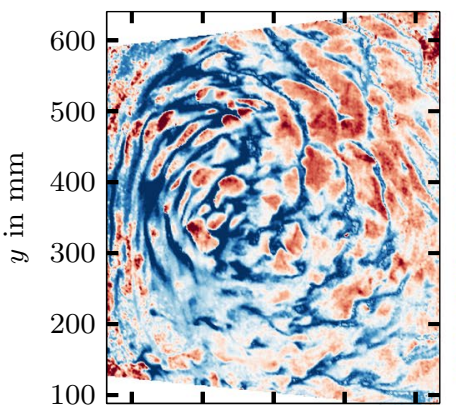

(a) $t=0 \mathrm{~s}(\tilde{t}=0)$

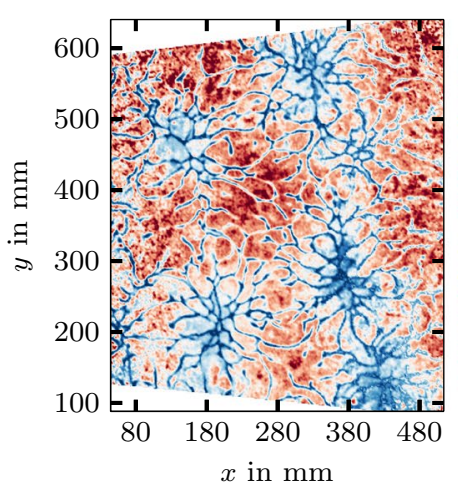

(e) $t=800 \mathrm{~s}(\tilde{t}=339.0)$

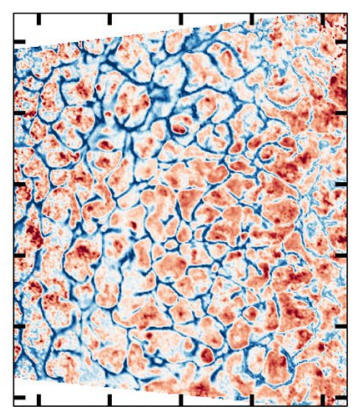

(b) $t=100 \mathrm{~s}(\tilde{t}=42.4)$

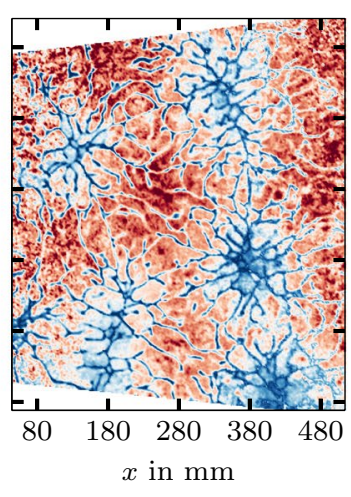

(f) $t=1100 \mathrm{~s}(\tilde{t}=466.1)$

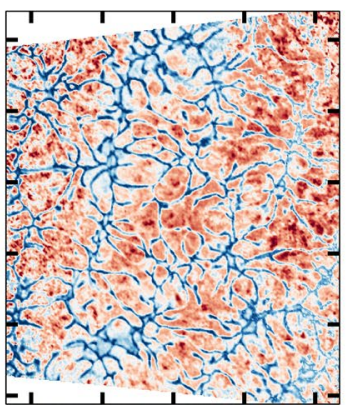

(c) $t=300 \mathrm{~s}(\tilde{t}=127.1)$

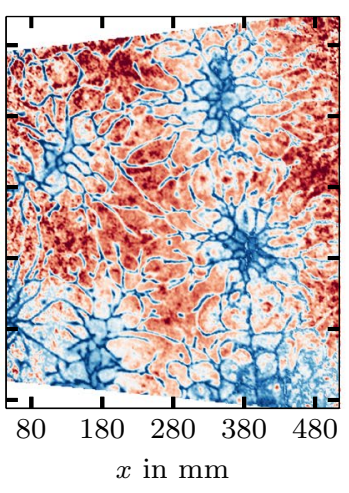

(g) $t=1400 \mathrm{~s}(\tilde{t}=593.2)$

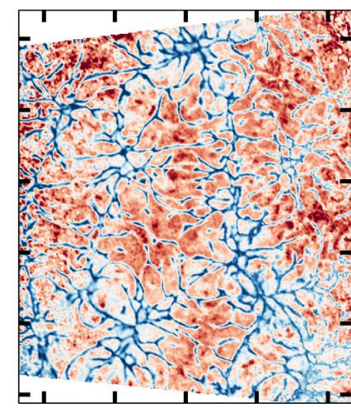

(d) $t=500 \mathrm{~s}(\tilde{t}=211.9)$

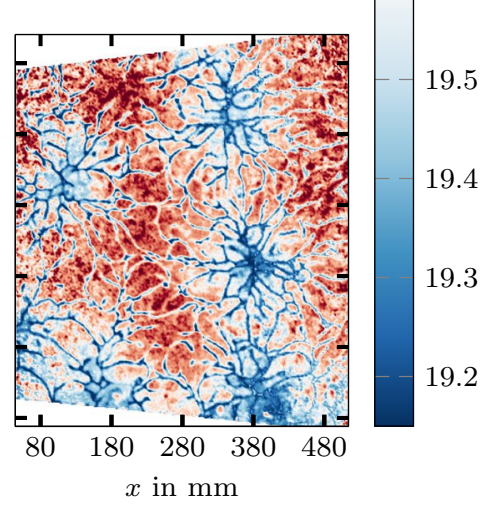

(h) $t=1700 \mathrm{~s}(\tilde{t}=720.3)$
20

19.8

○

$19.6 \stackrel{\circ}{.}$

E

9.5

19.3

19.2

Fig. 4 Instantaneous temperature fields for different instants of time, showing the initial growth of the flow structures subsequent to the end of the stirring process at $\tilde{t}=0$. The measurement was performed

in the top plane with nearly the same temperatures at the heating and cooling plate as in the long-time measurements, see Table 1 
transformed into Fourier space via a two-dimensional fast Fourier transformation (FFT), which was also used by Pandey et al. (2018) for the investigation of the length scales of turbulent superstructures. The resulting Fourier matrix was mapped to a polar wavevector grid, such that the Fourier coefficients on the single circumferences are related to the same wavenumber, but to varying orientations of the waves. Here, only the wavenumber is of interest, since this represents the size of the structures in the temperature field. Therefore, the Fourier coefficients, which are part of the single circumferences and describe the contribution of different wave orientations to the temperature field for one wavenumber, were summed up in azimuthal direction, respectively. Hence, the result is one value for each circumference, with the value representing the intensity of the single wavenumbers and, therefore, their contribution to the temperature field. For the illustration of the results in Fig. 5, the intensities are depicted in dependency of the wavelength $\lambda$, which is related to the wavenumber $k$ over $\lambda=2 \pi / k$. For a better comparability, the intensities are normalized with the maximum in each case. Furthermore, Fig. 5 shows the intensities starting from the instantaneous temperature field at $t=300$ $\mathrm{s}$, since temperature fields for earlier time instants are strongly affected by the initial stirring process. It can clearly be seen that the intensities of small wavelengths decrease over time, while the maximum intensity is shifted towards larger wavelengths. This demonstrates that small structures in the temperature field assemble to larger structures over time. The comparison of the results for the later points in time shows that the intensities are close together, meaning that the size of the structures does not change considerably anymore. Approaching the end of the investigated time range the maximum is mostly located at $\lambda=250 \pm 30 \mathrm{~mm}$. This wavelength also roughly matches the corresponding temperature fields in Fig. 4, since the mean distance of the most dominant patterns with similar temperature can be estimated to fall into this range. However, contrary to the wavelengths of the structures, their arrangement is gradually changing on the long run, as it will be shown in the following section.

\subsection{Extraction of turbulent superstructures}

By means of the instantaneous temperature fields obtained from the preliminary experiment presented in Sect. 3.1 the existence of turbulent superstructures could already be seen. However, in order to fully uncover the turbulent superstructures, the small-scale fluctuations must be removed by timeaveraging. In this context, the averaging time should fall into a certain range as suggested by Pandey et al. (2018). Following these suggestions, the small-scale fluctuations are removed, but the range of time-averaging is still small enough such that the turbulent superstructures are not smeared out or even completely removed. Accordingly, the recordings in the top and mid plane of the Rayleigh-Bénard cell were performed over a time range covering $t_{\text {rec }}=300 \mathrm{~s}$ in each case. Taking into account the free-fall times listed in Table 1, the recording time corresponds to $\tilde{t}_{\text {rec,top }} \approx 127 t_{\mathrm{f}}$ for the top plane measurements and to $\tilde{t}_{\text {rec,mid }} \approx 128 t_{\mathrm{f}}$ for the mid plane measurements. For all the measurements, the recording frequency was set to $f=5 \mathrm{~Hz}$, resulting in 1500 images, respectively. The effect of the time-averaging becomes obvious from Fig. 6, which shows an exemplary instantaneous temperature field from the top plane measurement and the corresponding field of the vertical velocity component as well as the temperature and velocity field averaged over the whole recording time of $127 t_{\mathrm{f}}$. Comparing the instantaneous and time-averaged fields it can be seen that the time-averaging removes the small-scale fluctuations with more extreme values of the temperature and velocity, resulting in clearly revealed turbulent superstructures. Furthermore, in particular the time-averaged fields illustrate that the occurring temperatures and velocities are well

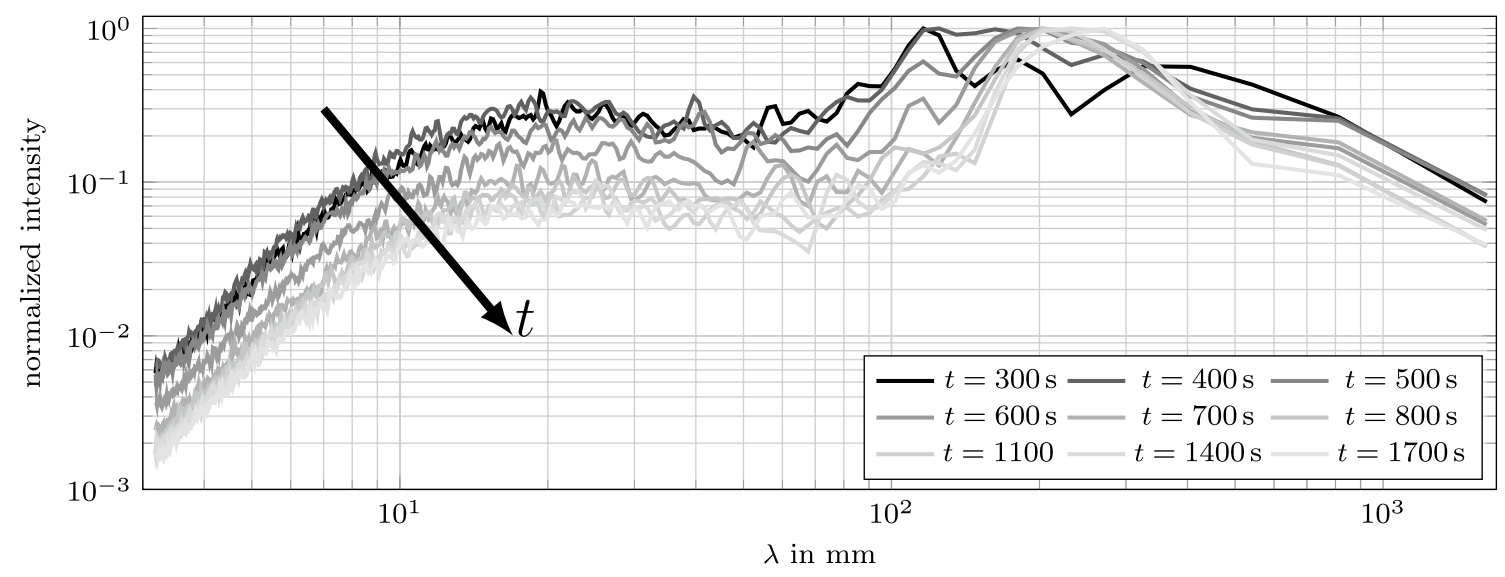

Fig. 5 Temporal variation of the normalized intensities of the wavelength spectrum obtained from the instantaneous temperature fields in Fig. 4, starting from $t=300 \mathrm{~s}$. In addition, the results for $t=400 \mathrm{~s}, t=600 \mathrm{~s}$ and $t=700 \mathrm{~s}$ are also included 


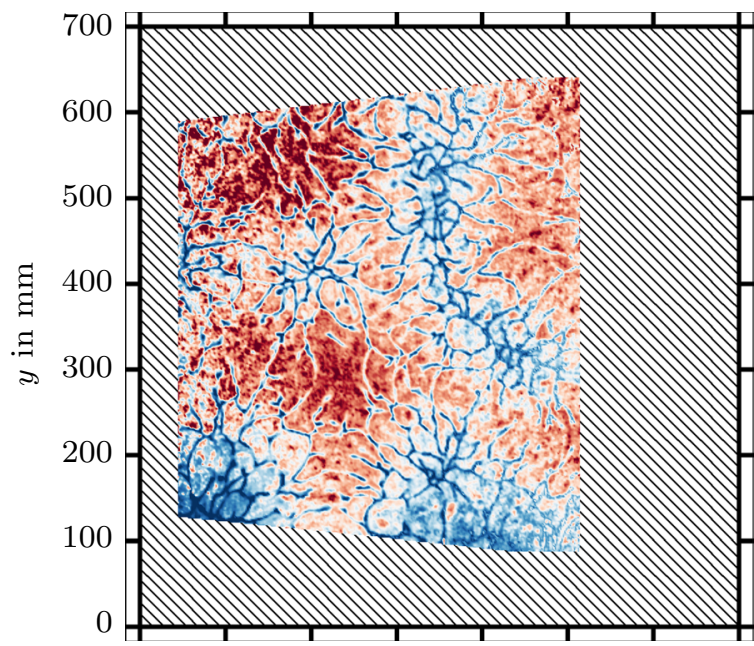

(a) Instantaneous temperature field

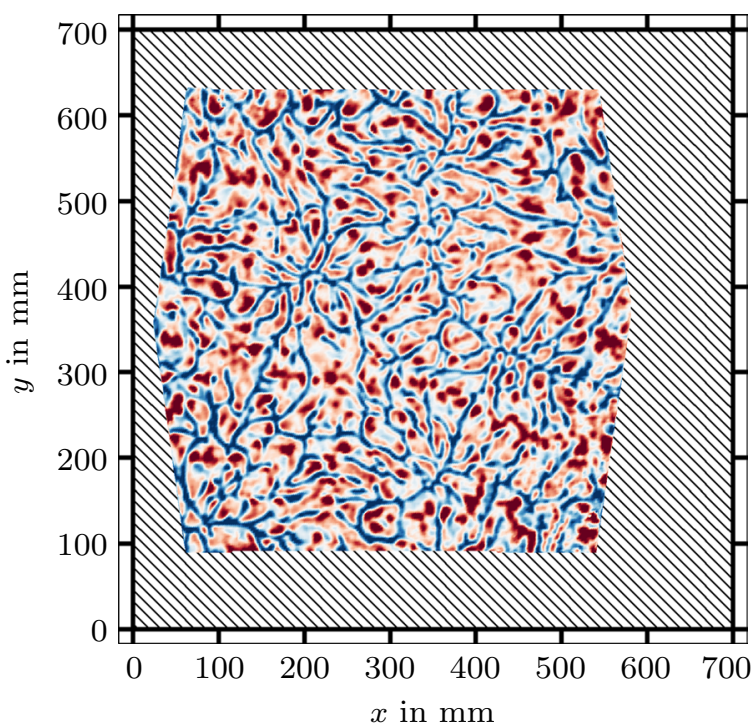

(c) Instantaneous field of the vertical velocity component

Fig. 6 Exemplary instantaneous fields of the temperature (a) and of the corresponding vertical velocity component (c) as well as the timeaveraged fields $(\mathbf{b}, \mathbf{d})$ obtained from a measurement in the top plane

correlated, since the distinctive warm and cold parts of the turbulent superstructures in the temperature field correspond to upwelling and downwelling fluid in the velocity field. For the sake of completeness it has to be noted that the temperature field is trapezoidal-shaped due to the observation angle of the color camera, while the velocity field covers the intersection of the field of view of the two monochrome cameras, which can be considered for the determination of the horizontal and vertical velocity components.

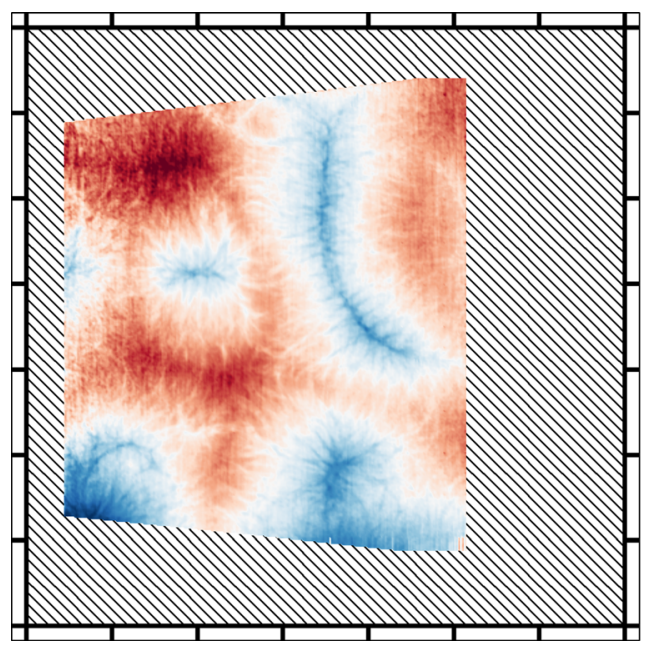

20

19.8

19.6. G

(b) Time-averaged temperature field

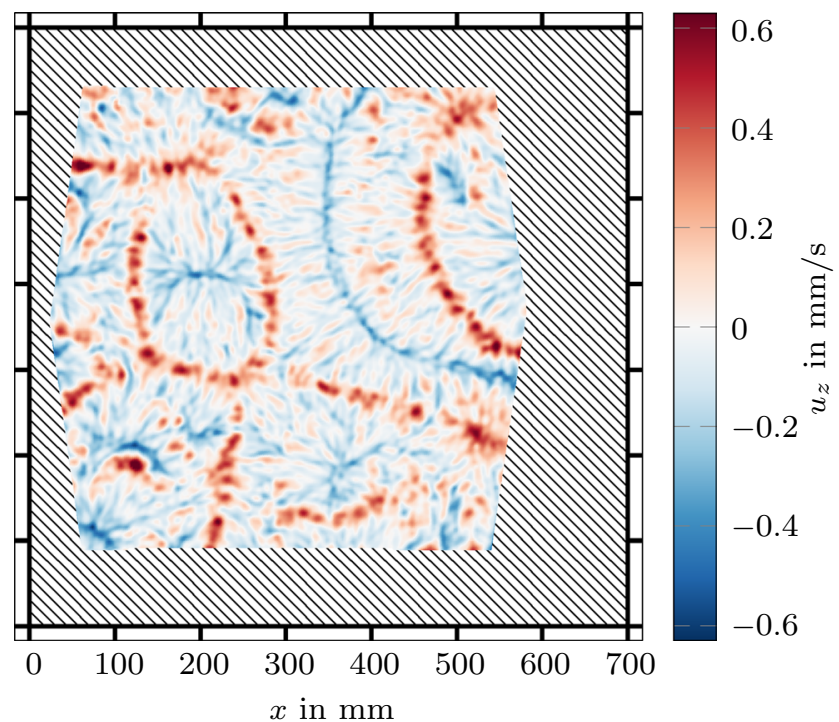

(d) Time-averaged field of the vertical velocity component

of the Rayleigh-Bénard cell according to Table 1. The time-averaging was performed over 1500 images covering about 127 free-fall times

\subsection{Reorganization of turbulent superstructures}

Aiming for deeper insights into the long-time behavior of turbulent superstructures, the experiments in both the top and mid plane were run over an extended time span of about $6 \mathrm{~h}$ and many series of 1500 images were recorded at different instants of time. In order to keep the focus for this study, two image series in each plane were processed and evaluated regarding the temperature and velocity fields, thereby enabling to show that the turbulent superstructures relocate over time. The time-averaged field of 
the temperature and of the vertical velocity component as well as of the absolute values of the horizontal velocities for the two exemplary image series of the measurements in the top and mid plane are depicted in Figs. 7 and 8 , respectively. For a better estimation of the results, the quantities are non-dimensionalized from here on, using the temperature difference $\Delta T=T_{\mathrm{h}}-T_{\mathrm{c}}$ for $\tilde{T}=T / \Delta T$, the free-fall velocity $u_{\mathrm{f}}=h / t_{\mathrm{f}}=\sqrt{\alpha g \Delta T h}$ for $\tilde{u}_{i}=u_{i} / u_{\mathrm{f}}$ and the height $h$ of the Rayleigh-Bénard cell for $\tilde{x}=x / h$, $\tilde{y}=y / h$ and $\tilde{z}=z / h$. Thus, the absolute value of the horizontal velocity is calculated by $\tilde{U}=\left(\tilde{u}_{x}^{2}+\tilde{u}_{y}^{2}\right)^{1 / 2}$. Furthermore, in Figs. 7 and 8 the beginning of the recording of the corresponding image series is given in free-fall times with the reference point $\tilde{t}=0$ at the end of the initial seeding process, during which the water in the cell is thoroughly stirred to achieve a homogeneous distribution of the TLCs. In order to allow for a better comparability, all the following results are restricted to the largest possible rectangular intersection of all the three cameras used for the recording. Hence, temperature and velocity data are available for the whole investigated section of the field of view. However, the vectors superimposed in the velocity fields are depicted with decreased resolution, using only each seventh grid point of the velocity field for illustration. It should also be noted that the length of the vectors was rescaled for a better illustration. Thus, the vectors do not represent the magnitude of the velocity, but only indicate the direction of the fluid motion in horizontal direction.

From Fig. 7 the turbulent superstructures in the top plane are clearly obvious in both the temperature and velocity

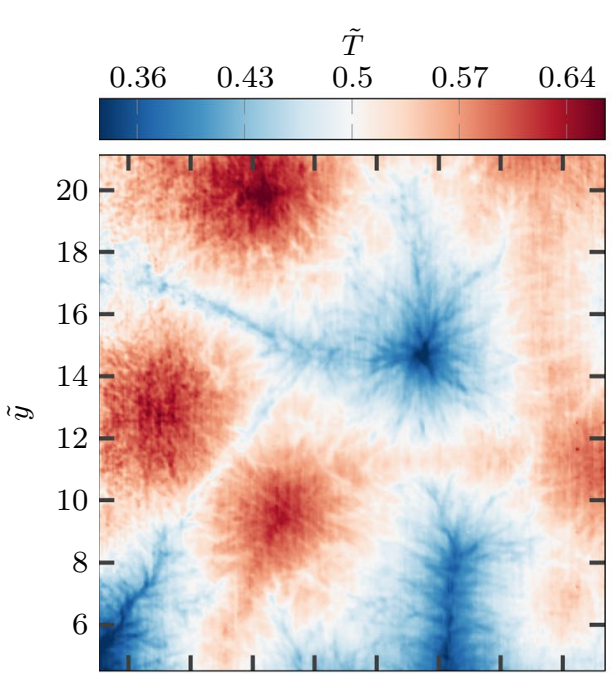

(a) $\left.\tilde{T}\right|_{\tilde{z}=0.86, \tilde{t}=4658.2}$

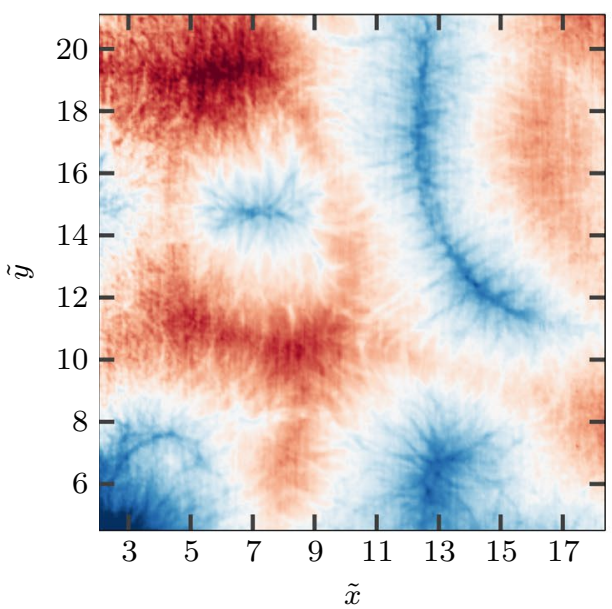

(d) $\left.\tilde{T}\right|_{\tilde{z}=0.86, \tilde{t}=6708.9}$

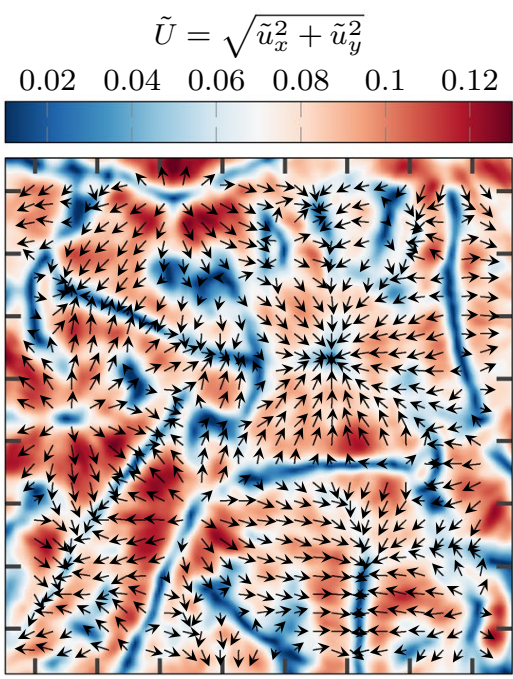

(b) $\left.\tilde{U}\right|_{\tilde{z}=0.86, \tilde{t}=4658.2}$

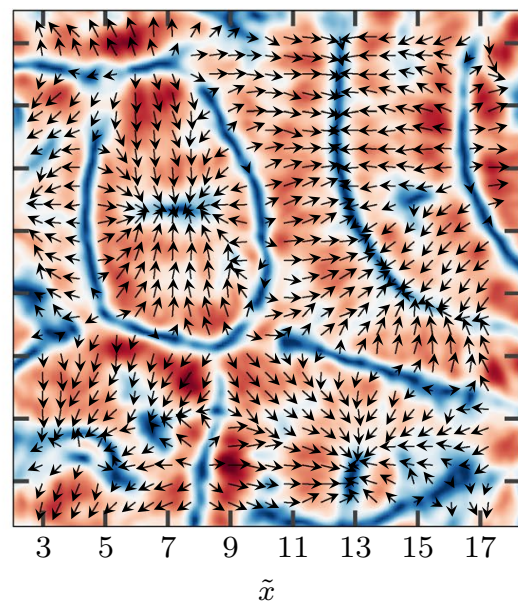

(e) $\left.\tilde{U}\right|_{\tilde{z}=0.86, \tilde{t}=6708.9}$

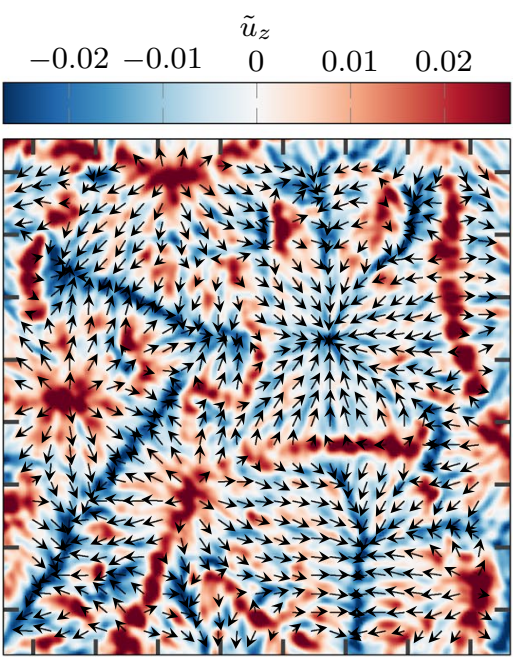

(c) $\left.\tilde{u}_{z}\right|_{\tilde{z}=0.86, \tilde{t}=4658.2}$

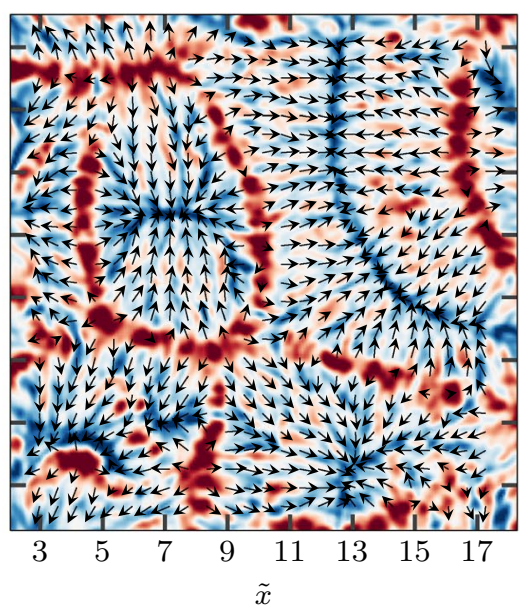

(f) $\left.\tilde{u}_{z}\right|_{\tilde{z}=0.86, \tilde{t}=6708.9}$
Fig. 7 Time averaged fields of the temperature (a, d), of the absolute value of the horizontal velocity $(\mathbf{b}, \mathbf{e})$ and of the vertical velocity component $(\mathbf{c}, \mathbf{f})$ showing the turbulent superstructures of the top plane measurement according to Table 1 for different time instants. The superimposed vectors in the middle and right column represent the direction of the horizontal velocity, respectively 


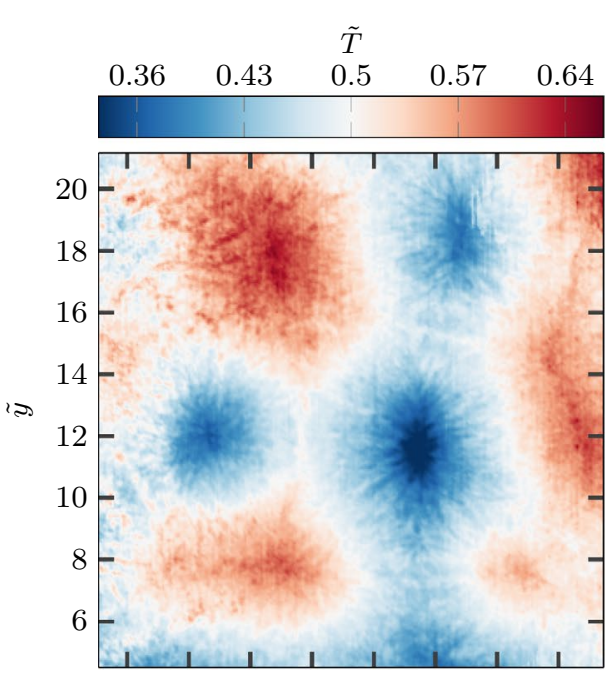

(a) $\left.\tilde{T}\right|_{\tilde{z}=0.5, \tilde{t}=1123.4}$

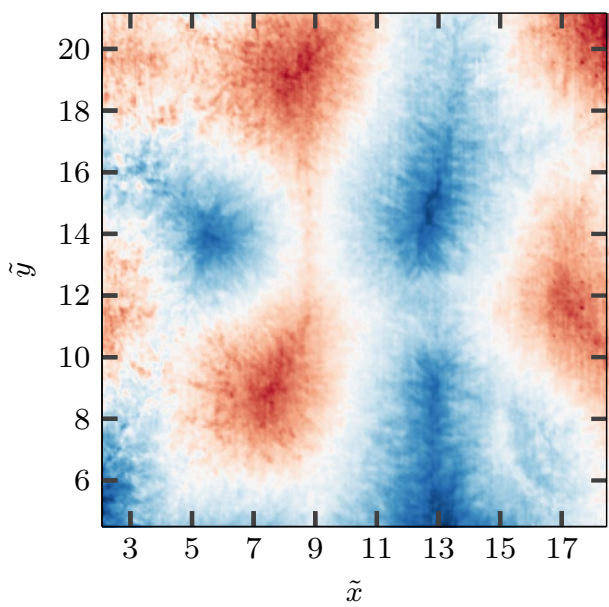

(d) $\left.\tilde{T}\right|_{\tilde{z}=0.5, \tilde{t}=2936.2}$

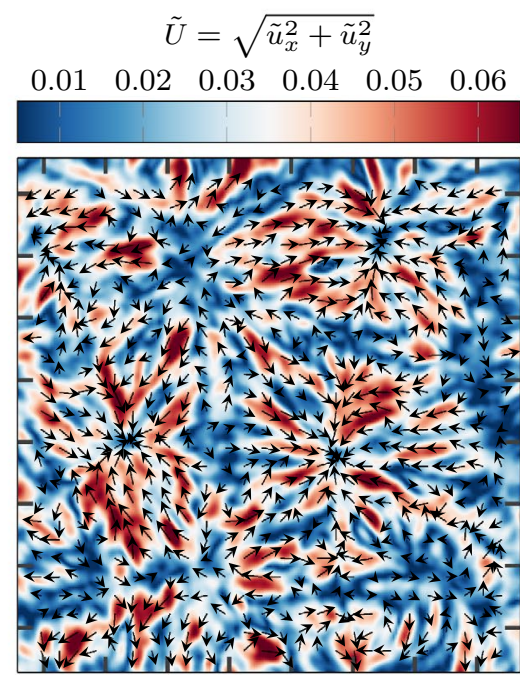

(b) $\left.\tilde{U}\right|_{\tilde{z}=0.5, \tilde{t}=1123.4}$

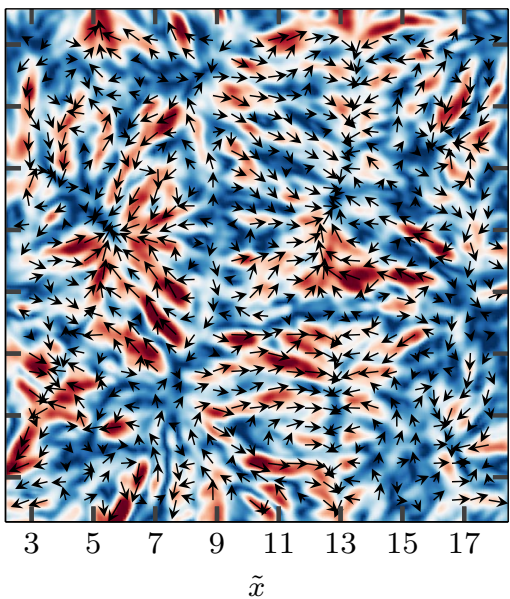

(e) $\left.\tilde{U}\right|_{\tilde{z}=0.5, \tilde{t}=2936.2}$

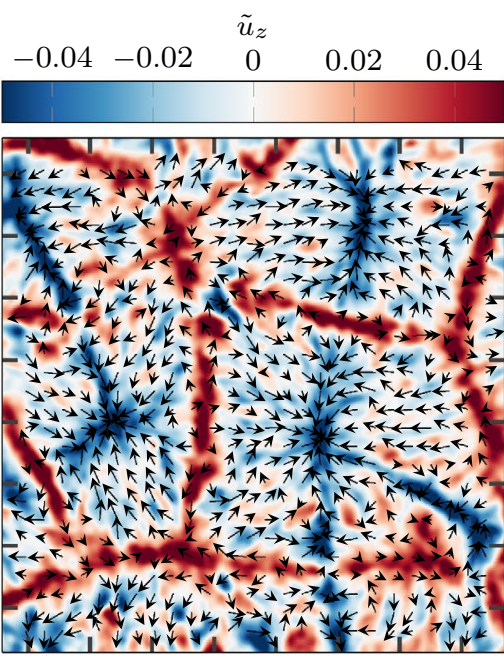

(c) $\left.\tilde{u}_{z}\right|_{\tilde{z}=0.5, \tilde{t}=1123.4}$

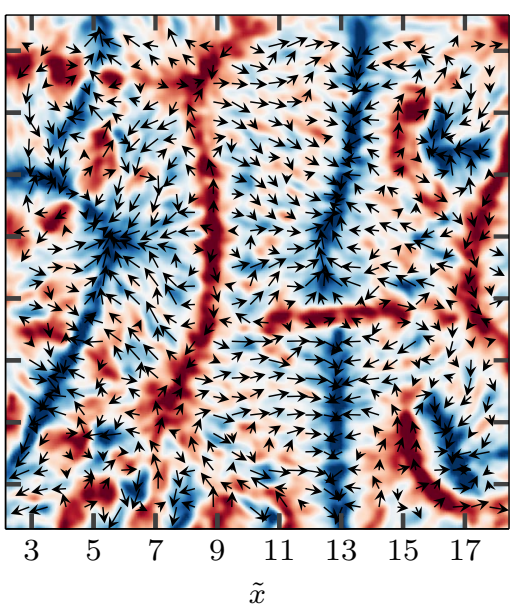

(f) $\left.\tilde{u}_{z}\right|_{\tilde{z}=0.5, \tilde{t}=2936.2}$
Fig. 8 Time averaged fields of the temperature (a, d), of the absolute value of the horizontal velocity $(\mathbf{b}, \mathbf{e})$ and of the vertical velocity component $(\mathbf{c}, \mathbf{f})$ showing the turbulent superstructures of the mid

fields. The comparison of the fields in the upper and lower row, which correspond to image series recorded with a time lag of about $2051 t_{\mathrm{f}}$ in this case, exemplarily shows that the turbulent superstructures rearrange over time. Furthermore, when viewing the fields along each of the rows, it can be seen that the temperature field matches the velocity field, respectively. As it can be expected, the warm parts of turbulent superstructures stream upward, while cold ones stream downward due to the variation of the fluid's density. In the central region of the warm and cold parts of the turbulent superstructures, where the most extreme temperatures occur, the absolute value of the vertical velocity is the highest and the horizontal velocity is the lowest. Analogously, the rearrangement of the turbulent superstructures over time and the plane measurement according to Table 1 for different time instants. The superimposed vectors in the middle and right column represent the direction of the horizontal velocity, respectively

correlation of the time-averaged temperature and velocity fields can also be found from the exemplary results of the mid plane measurements depicted in Fig. 8. Moreover, the measurements show that the range of the temperature in the mid plane is similar compared to that in the top plane, while the magnitude of the velocity components considerably differs between the planes, indicated by the colorbars at the top of each figure.

In order to specify the distributions of the temperature and velocity components in the top and mid plane more precisely, the corresponding probability density functions (PDFs) were calculated. For this, the spatially and temporally resolved temperature and velocity data of the two image series with 1500 images were considered, respectively. In 
Fig. 9 each value of a probability density $<10^{-3}$ was cut off, as the corresponding values for the velocity or temperature can be neglected due to their rareness and the main part of the PDFs is more distinguishable.

In Fig. 9a, b it can be seen that the horizontal velocity components $\tilde{u}_{x}$ and $\tilde{u}_{y}$ are similarly distributed in each case. However, the distribution of the horizontal velocities in the top plane is more flat and covers a larger range of about $\tilde{u}_{x}, \tilde{u}_{y} \in[-0.2,0.2]$, while the horizontal velocities in the mid plane are mainly in the range $\tilde{u}_{x}, \tilde{u}_{y} \in[-0.18,0.18]$. Furthermore, the differences concerning the vertical velocity component $\tilde{u}_{z}$ are very conspicuous, since the range of $\tilde{u}_{z} \in[-0.13,0.2]$ in the top plane is smaller and its distribution contains considerably more values around $\tilde{u}_{z}=0$ in comparison to the distribution of the vertical velocity component in the mid plane, which nearly matches that of the horizontal velocity components. Hence, in particular the distributions obtained from the mid plane confirm the three-dimensionality of the Rayleigh-Bénard flow at hand, since there is no dominant flow direction. On the contrary, in the top plane the flow is preferably directed into the $x$ - and $y$-direction, as the impacting, vertically moving fluid is redirected into the horizontal direction in close vicinity to the cooling plate. It can also be seen that the distribution of $\tilde{u}_{z}$ is more extended in positive $z$-direction, because the downward streaming fluid still accelerates within the top plane. The findings regarding the velocity components are quantitatively reflected in Table 2, in which the mean absolute value and the standard deviation of each component can be compared. In order to estimate the overall magnitude of the occurring velocities, the root-mean-square velocity $\tilde{U}_{\text {rms }}=\left(\left\langle\tilde{u}_{x}^{2}+\tilde{u}_{y}^{2}+\tilde{u}_{z}^{2}\right\rangle\right)^{1 / 2}$ incorporating the temporally and spatially resolved velocity components is listed in the table, too.

Furthermore, the PDFs in Fig. 9c represent the similarity of the temperature distribution in the top and mid plane. In both cases, temperatures in the range $\tilde{T} \in[0.25,0.75]$ are most likely to occur, with the probability density peaking around $\tilde{T}=0.5$ and thus around the mean of the heating and cooling plate temperature. Therefore, the average of the temperature of the top and mid plane measurements amounts to a similar value, listed in Table 2 . Even though this might not be intuitive, it is well-founded, since both measurement planes are not located within the thermal boundary layer (Scheel and Schumacher 2014) and, therefore, average

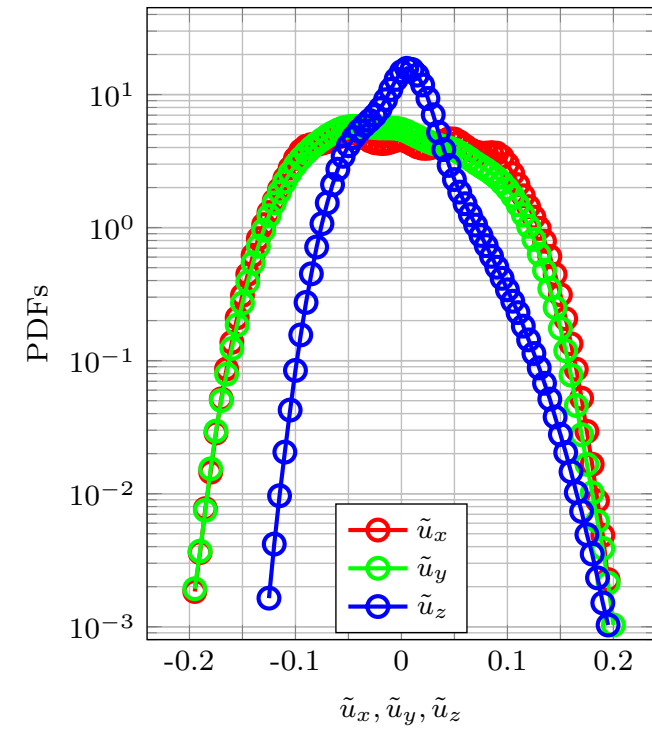

(a) Velocity components, top plane

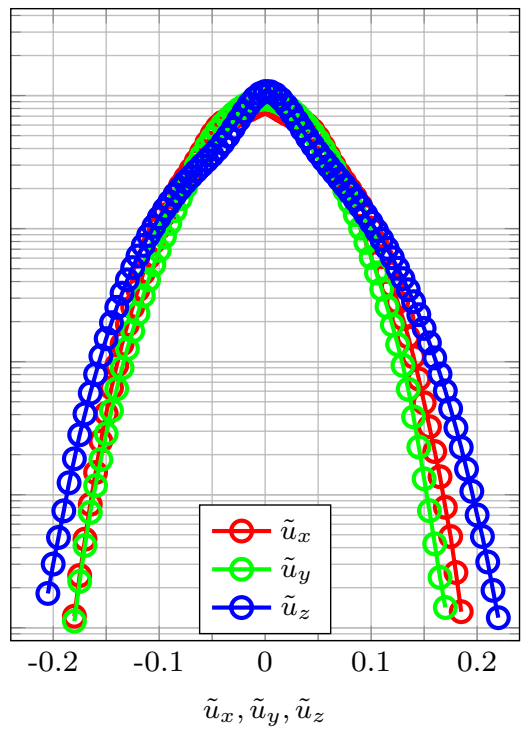

(b) Velocity components, mid plane

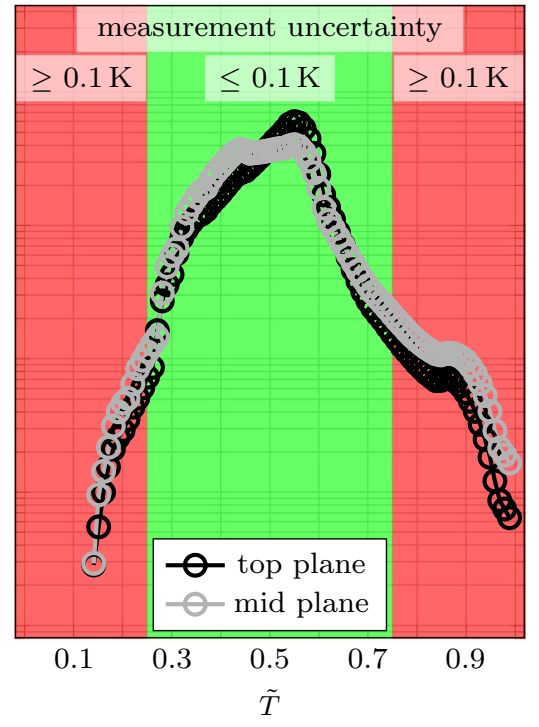

(c) Temperature

Fig. 9 Probability density functions (PDFs) of the three velocity components and of the temperature considering the full two data sets of the long-time measurements in the top and mid plane of the cell, respectively

Table 2 Mean absolute value and standard deviation of the velocity components and of the temperature for the whole data sets of the long-time measurements in the top and mid plane corresponding to the probability density functions shown in Fig. 9. Furthermore, the root-mean-square velocity $\tilde{U}_{\text {rms }}$ is listed for both measurement planes

\begin{tabular}{lllllllllll}
\hline Plane & $\tilde{z}$ & $\left\langle\left|\tilde{u}_{x}\right|\right\rangle$ & $\sigma_{\tilde{u}_{x}}$ & $\left\langle\left|\tilde{u}_{y}\right|\right\rangle$ & $\sigma_{\tilde{u}_{y}}$ & $\left\langle\left|\tilde{u}_{z}\right|\right\rangle$ & $\sigma_{\tilde{u}_{z}}$ & $\tilde{U}_{\text {rms }}$ & $\langle\tilde{T}\rangle$ & $\sigma_{\tilde{T}}$ \\
\hline Top & 0.86 & 0.059 & 0.069 & 0.053 & 0.063 & 0.025 & 0.033 & 0.100 & 0.512 & 0.095 \\
Mid & 0.50 & 0.038 & 0.048 & 0.034 & 0.043 & 0.038 & 0.050 & 0.081 & 0.495 & 0.108 \\
\hline
\end{tabular}


temperatures around $\tilde{T}=0.5$ can be expected. Looking at temperatures of $\tilde{T}<0.25$ and $\tilde{T}>0.75$, it can be found from Fig. $9 \mathrm{c}$ that the probability density does not decrease symmetrically. However, the results for these temperature ranges should not be interpreted physically, as the settings of the temperature measurement technique were optimized to work most robustly in the range $\tilde{T} \in[0.25,0.75]$. In this range that covers most of the occurring temperatures, the uncertainty of measurement does not exceed $0.1 \mathrm{~K}$, also indicated in Fig. 9c. In this context it is noted that the probability density functions in Fig. 9c are plotted logarithmically on the axis of ordinate, ranging over more than three orders of magnitude from the minimum to the maximum values, which confirms the predominance of the central temperature range. In addition, this is also affirmed by the standard deviations of the temperature distributions listed in the last column of Table 2.

\subsection{Analysis of the local heat flux}

One of the most important benefits of simultaneous temperature and velocity fields is to use these data for the evaluation of the local heat flux in Rayleigh-Bénard convection (Shang et al. 2004; Shishkina and Wagner 2007). Here, this possibility is demonstrated by means of an exemplary measurement in the mid plane of the Rayleigh-Bénard cell.

In comparison to the state, where the fluid in the cell is at rest and heat is only transported via conduction for small temperature differences between the heating and cooling plate, the heat flux is strongly enhanced when convective motion sets in. With increasing temperature difference between the isothermal plates and accordingly also increasing Rayleigh number, the heat flux is more and more dominated by convection, which can be quantified by the so-called Nusselt number $\mathrm{Nu}$. This dimensionless number represents the ratio between the total heat flux from the bottom to the top plate and the heat flux solely due to heat conduction between these plates, yielding $\mathrm{Nu}=\dot{Q} / \dot{Q}_{\text {cond. }}$. This ratio can also be determined locally based on the local temperature and the vertical velocity component, thereby obtaining the local Nusselt number $\mathrm{Nu}_{\mathrm{loc}}=1+\sqrt{\operatorname{Ra} \operatorname{Pr}} \tilde{u}_{z} \Theta$ (Pandey et al. 2018; Fonda et al. 2019). The last variable $\Theta$ in this equation denotes the dimensionless temperature difference between the local temperature $\tilde{T}(x, y)$ and the temperature that would result without convective motion at the height of the measurement plane, i.e. $\Theta=\tilde{T}(x, y)-(1-\tilde{z})$. In order to apply this equation to the measurement results presented in this study, the temperature and velocity information must be available for the same grid points. Therefore, the velocity field with the slightly coarser grid resolution given in Table 1 was determined for the grid of the temperature field via nearest neighbor interpolation. On this grid the field of the local Nusselt number was exemplarily computed for the instantaneous temperature and velocity fields of the first measurement series in the mid plane, which were time-averaged for the results shown in Fig. 8a-c. The time-averaging of the local Nusselt number yields the field shown in Fig. 10c, directly opposed to the time-averaged fields of the temperature and the vertical velocity component in Fig. 10a, b for a better comparison. The influence of the turbulent superstructures on the local heat flux becomes clearly apparent from Fig. 10. In this figure it can be seen that the local Nusselt number reaches the highest values in the regions with the dominant updrafts and downdrafts, confirming that turbulent superstructures transport the heat most effectively. From Fig. 10c it is obvious that the local Nusselt number may also have a negative value, resulting from either a warm fluid parcel streaming downward or a cold one streaming upward. Even though this is not expected with regard to the change of the fluid's density, it can appear locally due to the viscous friction at the adjacent fluid parcels. However, the positive values of the local Nusselt number predominate, meaning that warm fluid is mainly streaming upward, while cold fluid is streaming downward. The predominance of the positive values of the local Nusselt number is confirmed by its average, yielding the global Nusselt number $\mathrm{Nu}_{\mathrm{glob}} \approx 6.5$ in this case.

\subsection{Comparison with numerical results}

In order to assess the presented experimental results, those are shortly compared to the results of numerical simulations. For example, Fonda et al. (2019) have performed simulations of Rayleigh-Bénard convection for the same aspect ratio and Prandtl number, i.e. $\Gamma=25$ and $\operatorname{Pr}=7$, for three different Rayleigh numbers of $\mathrm{Ra}_{1}=10^{5}, \mathrm{Ra}_{2}=10^{6}$ as well as $\mathrm{Ra}_{3}=10^{7}$ and obtained global Nusselt numbers of $\mathrm{Nu}_{\mathrm{glob}, 1}=4.1, \mathrm{Nu}_{\mathrm{glob}, 2}=8.3$ and $\mathrm{Nu}_{\mathrm{glob}, 3}=16.2$. Applying the exponential fit $\mathrm{Nu}_{\text {glob }}=0.133 \mathrm{Ra}^{0.298}$ from these three data points to the Rayleigh number of $\mathrm{Ra}=7.65 \times 10^{5}$ of the presented measurement in the mid plane, which was analyzed with regard to the local Nusselt number in the Sect. 3.4, gives $\mathrm{Nu}_{\text {glob }} \approx 7.6$. Hence, the global Nusselt number which can be estimated on the basis of those numerical data is slightly larger than the Nusselt number $\mathrm{Nu}_{\mathrm{glob}} \approx 6.5$ determined from the simultaneous temperature and velocity field measurements in this experimental study.

Another important aspect for the comparison of the experimental and numerical results is the wavelength of the turbulent superstructures. A one-to-one comparison is not possible so far, as the Rayleigh numbers of the experiments do not directly match the Rayleigh numbers of previous numerical simulations. Performing measurements, which are directly comparable to numerical simulations, will be the focus of the future work, however, a rough comparison is already possible. The wavelength of turbulent superstructures in water was especially investigated by 


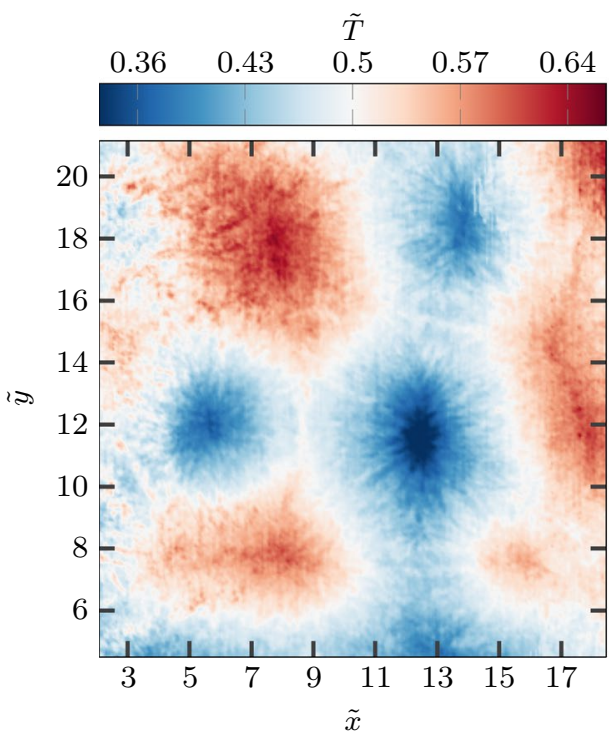

(a) $\left.\tilde{T}\right|_{\tilde{z}=0.5}$

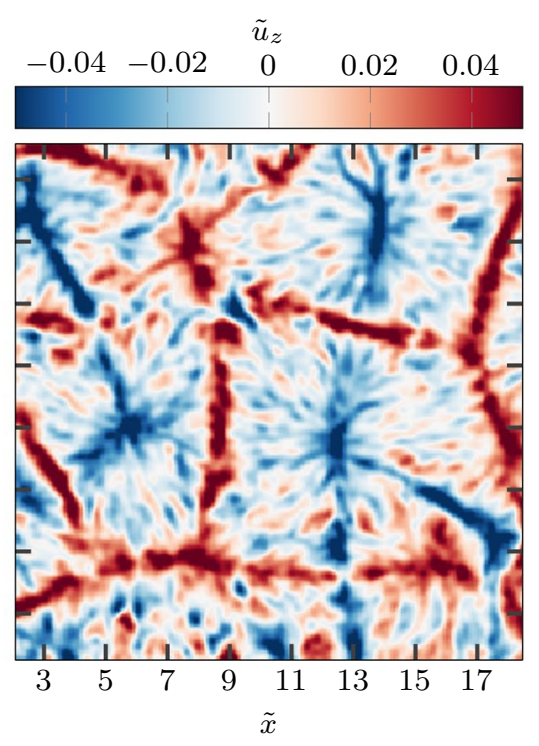

(b) $\tilde{u}_{z} \mid \tilde{z}=0.5$

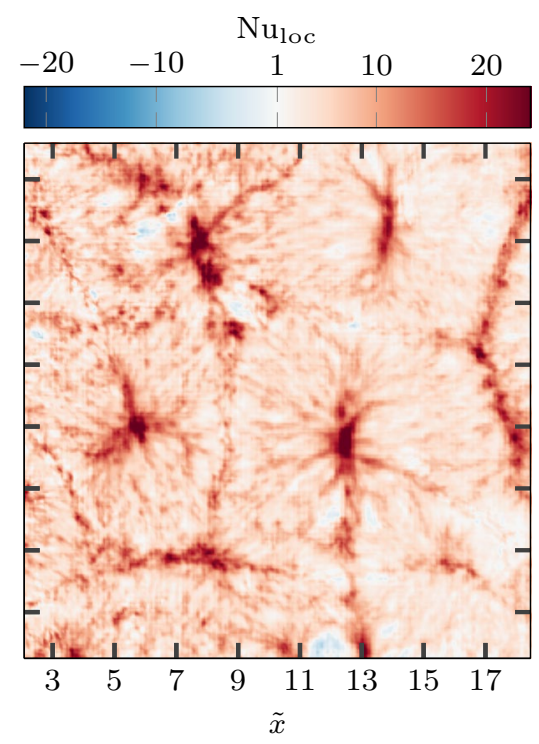

(c) $\mathrm{Nu}_{\text {loc }} \mid \tilde{z}=0.5$

Fig. 10 Comparison of the time-averaged fields of the temperature (a), the vertical velocity component (b) and the local Nusselt number (c) obtained from an exemplary measurement set in the mid plane of the Rayleigh-Bénard cell. The averaging time is $128 t_{\mathrm{f}}$ in each case

Fonda et al. (2019). For the size of the turbulent superstructures in the temperature field $\lambda_{T}$ and in the vertical velocity field $\lambda_{W}$, they obtained $\lambda_{T}=6.3$ and $\lambda_{W}=5.4$ for $\mathrm{Ra}=10^{5}$ as well as $\lambda_{T}=6.3$ and $\lambda_{W}=5.7$ for $\mathrm{Ra}=10^{6}$ in the mid plane of the cell. Hence, for the comparison the exemplary results of the mid plane measurement with the intermediate Rayleigh number of $\mathrm{Ra}=7.65 \times 10^{5}$ depicted in Fig. 8a-c were considered. Using the two-dimensional Fourier analysis outlined in Sect. 3.1 for the investigation of the wavelength in this set of the mid plane measurement yields $\lambda_{T}=9.7$ and $\lambda_{W}=8.3$. Therefore, in this experimental study the wavelength of the turbulent superstructures is larger in both the temperature and the velocity field.

Furthermore, a good measure for the comparison of the experimental and numerical data is the evaluation of the root-mean-square velocity. This velocity is also given in the study of Fonda et al. (2019) and ranges in between $\tilde{U}_{\text {rms }} \in[0.089,0.102]$ for the different runs of the simulations for the Rayleigh numbers $\mathrm{Ra}=10^{5}$ and $\mathrm{Ra}=10^{6}$. Thus, the results for the root-mean-square velocity in Table 2 obtained from the presented measurements in the mid plane and top plane show a good agreement with the numerical results.

\section{Conclusion}

In this study turbulent superstructures in Rayleigh-Bénard convection were investigated experimentally. The experiments were performed in the horizontal mid plane and in a horizontal plane closer to the cooling plate at the top of a Rayleigh-Bénard cell with an aspect ratio of $\Gamma=25$. In an additional preliminary experiment the initial growth of the flow structures was shown by means of temperature measurements via the color signal of thermochromic liquid crystals (TLCs) in the top measurement plane. Based on this experiment it could be seen that small structures in instantaneous temperature fields assemble over time, resulting in larger structures, which already indicate the existence of turbulent superstructures. However, the main goal of this study was to fully uncover and investigate those larger structures, which requires a time-averaging of the temperature and velocity fields. Hence, time-averaged temperature and velocity fields were determined by evaluating the color signal of TLCs for the temperature measurements and their motion for simultaneous velocity measurements. While the color signal was recorded with a single color camera, the motion was recorded with two monochrome cameras, such that all three velocity components in the horizontal planes could be measured via stereoscopic particle image velocimetry, respectively.

For both the top and mid plane measurement an image series of the TLCs was recorded for two different time instants. In each case, the time-averaging of the instantaneous temperature and velocity fields removed the small-scale fluctuations, thereby clearly revealing the turbulent superstructures. The comparison of the results for the two image series, respectively, showed that the turbulent superstructures rearrange over time. Furthermore, the time-averaged temperature fields and the corresponding velocity fields 
were well correlated in each case, meaning that the contours of the turbulent superstructures in the temperature field nearly coincide with those of the velocity field. Moreover, comparing the time-averaged fields of the top and mid plane measurements it could be seen that the turbulent superstructures cover similar ranges regarding the temperature, but considerably differing ranges regarding the velocity components. This was quantitatively confirmed by means of probability density functions (PDFs) for all the velocity components and the temperature, which incorporate the whole two data sets of the spatially and temporally resolved measurements in the top and mid plane, respectively.

In addition, one of the main advantages of the simultaneous measurements of temperature and velocity fields was demonstrated in this study. The combination of the temperature and the vertical velocity component was utilized to characterize the local heat transport in Rayleigh-Bénard convection by the local Nusselt number. In this context it was shown that the turbulent superstructures are of vital importance for the heat transport from the heating to the cooling plate of the cell, since distinctive local maxima of the Nusselt number appear at the position of these structures.

The results obtained from this experimental study were also compared to the outcomes of numerical simulations regarding the global heat transport, the wavelength of the turbulent superstructures and the overall magnitude of the velocity. While the global Nusselt number, which represents the total amount of heat transport, and the magnitude of the velocity in terms of the root-mean-square velocity have shown a good agreement, the wavelength of the turbulent superstructures in the temperature and the velocity field is smaller according to the numerical results. Considering that the experimental and numerical approach for the investigation of the flow have their own specific strengths as well as drawbacks, some differences of the results can also be expected. However, all in all it can be concluded that the results are consistent, such that the applicability of both the experimental and numerical methods is confirmed.

Having built up this experiment, it is now possible to investigate turbulent superstructures in Rayleigh-Bénard convection in greater detail. It is highlighted again that this setup enables optical access to the full horizontal crosssectional area of $l \times w=700 \mathrm{~mm} \times 700 \mathrm{~mm}$. Even though the side walls of the cell are not expected to considerably affect the flow in large aspect ratio cells, the investigated cross-sectional area was restricted to a central section with dimensions of about $l_{x} \times l_{y}=460 \mathrm{~mm} \times 470 \mathrm{~mm}$ in this case, thereby increasing the spatial resolution of the measurement. Furthermore, this setup allows to study the flow over extended time spans of several hours, in order to gain deeper insights into the long-time rearrangement of turbulent superstructures. In particular, the latter feature is an important complement to studies based on numerical simulations, which are commonly performed with a high spatial resolution, but do not cover such large time spans up to date.

\section{Outlook}

In future experiments especially the effect of the Rayleigh number on the turbulent superstructures will be investigated by applying different temperature differences between the heating and cooling plate, thereby enabling a more comprehensive comparison of the experimental and numerical results. Furthermore, also the aspect ratio of the Rayleigh-Bénard cell will be varied to analyze the influence of the cell's geometry on turbulent superstructures. On the basis of the experimental data, the focus of the future work will be on the analysis of the long-time reorganization of turbulent superstructures, thereby determining typical time scales for their modification. Moreover, besides their temporal scales also the spatial scaling as well as their impact on the local and global transport of heat and momentum will be studied in greater detail, yielding a better understanding of the flow in large aspect ratio Rayleigh-Bénard setups.

Acknowledgements The authors would like to thank Jens Fokken and Alexander Thieme for the technical support during the measurements and Ambrish Pandey as well as Jörg Schumacher for valuable discussions. Furthermore, the authors acknowledge support from the German Research Foundation (Deutsche Forschungsgemeinschaft, DFG) within the Priority Programme Turbulent Superstructures SPP 1881.

Funding Open Access funding enabled and organized by Projekt DEAL.

Open Access This article is licensed under a Creative Commons Attribution 4.0 International License, which permits use, sharing, adaptation, distribution and reproduction in any medium or format, as long as you give appropriate credit to the original author(s) and the source, provide a link to the Creative Commons licence, and indicate if changes were made. The images or other third party material in this article are included in the article's Creative Commons licence, unless indicated otherwise in a credit line to the material. If material is not included in the article's Creative Commons licence and your intended use is not permitted by statutory regulation or exceeds the permitted use, you will need to obtain permission directly from the copyright holder. To view a copy of this licence, visit http://creativecommons.org/licenses/by/4.0/.

\section{References}

Bailon-Cuba J, Emran MS, Schumacher J (2010) Aspect ratio dependence of heat transfer and large-scale flow in turbulent convection. J Fluid Mech 655:152-173. https://doi.org/10.1017/s002211201 0000820

Bodenschatz E, Pesch W, Ahlers G (2000) Recent developments in Rayleigh-Bénard convection. Annu Rev Fluid Mech 32(1):709778. https://doi.org/10.1146/annurev.fluid.32.1.709

Burr U, Müller U (2001) Rayleigh-Bénard convection in liquid metal layers under the influence of a vertical magnetic field. Phys Fluids 13(11):3247-3257. https://doi.org/10.1063/1.1404385 
Cierpka C, Kästner C, Resagk C, Schumacher J (2019) On the challenges for reliable measurements of convection in large aspect ratio Rayleigh-Bénard cells in air and sulfur-hexafluoride. Exp Thermal Fluid Sci 109:109841. https://doi.org/10.1016/j.expth ermflusci.2019.109841

Cramer A, Pal J, Gerbeth G (2013) Model experiments for the Czochralski crystal growth technique. Eur Phys J Spec Top 220(1):259-273. https://doi.org/10.1140/epjst/e2013-01812-5

Dabiri D (2008) Digital particle image thermometry/velocimetry: a review. Exp Fluids 46(2):191-241. https://doi.org/10.1007/s0034 8-008-0590-5

du Puits R, Resagk C, Thess A (2007) Breakdown of wind in turbulent thermal convection. Phys Rev E. https://doi.org/10.1103/ physreve.75.016302

Emran MS, Schumacher J (2008) Fine-scale statistics of temperature and its derivatives in convective turbulence. J Fluid Mech 611:1334. https://doi.org/10.1017/s0022112008002954

Emran MS, Schumacher J (2015) Large-scale mean patterns in turbulent convection. J Fluid Mech 776:96-108. https://doi. org/10.1017/jfm.2015.316

Fonda E, Pandey A, Schumacher J, Sreenivasan KR (2019) Deep learning in turbulent convection networks. Proc Natl Acad Sci 116(18):8667-8672. https://doi.org/10.1073/pnas.1900358116

Green G, Vlaykov DG, Mellado JP, Wilczek M (2020) Resolved energy budget of superstructures in Rayleigh-Bénard convection. J Fluid Mech. https://doi.org/10.1017/jfm.2019.1008

Hartlep T, Tilgner A, Busse FH (2003) Large scale structures in Rayleigh-Bénard convection at high Rayleigh numbers. Phys Rev Lett. https://doi.org/10.1103/physrevlett.91.064501

Hartlep T, Tilgner A, Busse FH (2005) Transition to turbulent convection in a fluid layer heated from below at moderate aspect ratio. J Fluid Mech 544(1):309. https://doi.org/10.1017/s0022112005006671

Kästner C, Resagk C, Westphalen J, Junghähnel M, Cierpka C, Schumacher J (2018) Assessment of horizontal velocity fields in square thermal convection cells with large aspect ratio. Exp Fluids. https ://doi.org/10.1007/s00348-018-2626-9

Krug D, Lohse D, Stevens RJAM (2020) Coherence of temperature and velocity superstructures in turbulent Rayleigh-Bénard flow. J Fluid Mech. https://doi.org/10.1017/jfm.2019.1054

Lohse D, Xia KQ (2010) Small-scale properties of turbulent RayleighBénard convection. Annu Rev Fluid Mech 42(1):335-364. https ://doi.org/10.1146/annurev.fluid.010908.165152

Mishra PK, De AK, Verma MK, Eswaran V (2010) Dynamics of reorientations and reversals of large-scale flow in Rayleigh-Bénard convection. J Fluid Mech 668:480-499. https://doi.org/10.1017/ s0022112010004830

Moller S, König J, Resagk C, Cierpka C (2019) Influence of the illumination spectrum and observation angle on temperature measurements using thermochromic liquid crystals. Meas Sci Technol 30(8):084006. https://doi.org/10.1088/1361-6501/ab173f

Moller S, Resagk C, Cierpka C (2020) On the application of neural networks for temperature field measurements using thermochromic liquid crystals. Exp Fluids. https://doi.org/10.1007/s00348-020-2943-7

Pandey A, Scheel JD, Schumacher J (2018) Turbulent superstructures in Rayleigh-Bénard convection. Nat Commun. https://doi. org/10.1038/s41467-018-04478-0

Raffel M, Willert CE, Scarano F, Kähler CJ, Wereley ST, Kompenhans J (2018) Particle image velocimetry. Springer International
Publishing, New York. https://doi.org/10.1007/978-3-319-68852 $-7$

Sakievich P, Peet Y, Adrian R (2016) Large-scale thermal motions of turbulent Rayleigh-Bénard convection in a wide aspect-ratio cylindrical domain. Int J Heat Fluid Flow 61:183-196. https://doi. org/10.1016/j.ijheatfluidflow.2016.04.011

Scheel JD, Schumacher J (2014) Local boundary layer scales in turbulent Rayleigh-Bénard convection. J Fluid Mech 758:344-373. https://doi.org/10.1017/jfm.2014.536

Scheel JD, Emran MS, Schumacher J (2013) Resolving the fine-scale structure in turbulent Rayleigh-Bénard convection. New J Phys 15(11):113063. https://doi.org/10.1088/1367-2630/15/11/113063

Schmeling D, Bosbach J, Wagner C (2014) Simultaneous measurement of temperature and velocity fields in convective air flows. Meas Sci Technol 25(3):035302. https://doi.org/10.1088/09570233/25/3/035302

Schneide C, Pandey A, Padberg-Gehle K, Schumacher J (2018) Probing turbulent superstructures in Rayleigh-Bénard convection by Lagrangian trajectory clusters. Phys Rev Fluids. https://doi. org/10.1103/physrevfluids.3.113501

Schumacher J, Sreenivasan KR (2020) Colloquium: unusual dynamics of convection in the sun. Rev Mod Phys. https://doi.org/10.1103/ revmodphys.92.041001

Shang XD, Qiu XL, Tong P, Xia KQ (2004) Measurements of the local convective heat flux in turbulent Rayleigh-Bénard convection. Phys Rev E. https://doi.org/10.1103/physreve.70.026308

Shishkina O, Wagner C (2007) Local heat fluxes in turbulent Rayleigh-Bénard convection. Phys Fluids 19(8):085107. https://doi. org/10.1063/1.2756583

Stevens RJAM, Blass A, Zhu X, Verzicco R, Lohse D (2018) Turbulent thermal superstructures in Rayleigh-Bénard convection. Phys Rev Fluids. https://doi.org/10.1103/physrevfluids.3.041501

van der Poel EP, Stevens RJAM, Sugiyama K, Lohse D (2012) Flow states in two-dimensional Rayleigh-Bénard convection as a function of aspect-ratio and Rayleigh number. Phys Fluids 24(8):085104. https://doi.org/10.1063/1.4744988

von Hardenberg J, Parodi A, Passoni G, Provenzale A, Spiegel E (2008) Large-scale patterns in Rayleigh-Bénard convection. Phys Lett A 372(13):2223-2229. https://doi.org/10.1016/j.phys] eta.2007.10.099

Wieneke B (2005) Stereo-PIV using self-calibration on particle images. Exp Fluids 39(2):267-280. https://doi.org/10.1007/s0034 8-005-0962-z

Zhang Y, Li J, Liu M, Liu J, Wang C (2020) Experimental investigation of large-scale flow structures in an aircraft cabin mockup. Build Environ 184:107224. https://doi.org/10.1016/j.build env.2020.107224

Zhou Q, Xi HD, Zhou SQ, Sun C, Xia KQ (2009) Oscillations of the large-scale circulation in turbulent Rayleigh-Bénard convection: the sloshing mode and its relationship with the torsional mode. J Fluid Mech 630:367-390. https://doi.org/10.1017/s0022112009006764

Publisher's Note Springer Nature remains neutral with regard to jurisdictional claims in published maps and institutional affiliations. 


\section{Authors and Affiliations}

\section{Sebastian Moller ${ }^{1}\left[\right.$ Christian Resagk $^{1} \cdot$ Christian Cierpka $^{1}$}

Institute of Thermodynamics and Fluid Mechanics,

Technische Universität Ilmenau, Am Helmholtzring 1,

98693 Ilmenau, Germany 